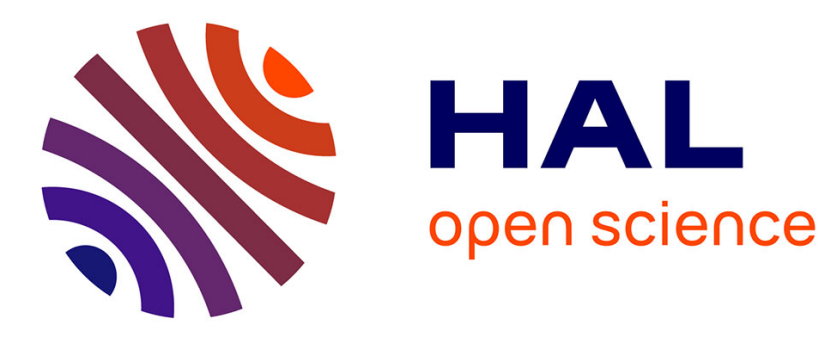

\title{
A Social-aware Routing Protocol for Opportunistic Networks
}

Ana Cristina Kochem Vendramin, Anelise Munaretto, Myriam Regattieri Delgado, Aline Carneiro Viana, Mauro Fonseca

\section{- To cite this version:}

Ana Cristina Kochem Vendramin, Anelise Munaretto, Myriam Regattieri Delgado, Aline Carneiro Viana, Mauro Fonseca. A Social-aware Routing Protocol for Opportunistic Networks. Expert Systems with Applications, 2016, 34, pp.351-363. 10.1016/j.eswa.2016.01.039 . hal-01369964

\section{HAL Id: hal-01369964 https://hal.science/hal-01369964}

Submitted on 17 Oct 2016

HAL is a multi-disciplinary open access archive for the deposit and dissemination of scientific research documents, whether they are published or not. The documents may come from teaching and research institutions in France or abroad, or from public or private research centers.
L'archive ouverte pluridisciplinaire HAL, est destinée au dépôt et à la diffusion de documents scientifiques de niveau recherche, publiés ou non, émanant des établissements d'enseignement et de recherche français ou étrangers, des laboratoires publics ou privés. 


\title{
A Social-Aware Routing Protocol For Opportunistic Networks
}

\author{
Ana Cristina Kochem Vendramin ${ }^{\mathrm{a}, 1}$, Anelise Munaretto ${ }^{\mathrm{a}}$, Myriam \\ Regattieri Delgado ${ }^{\mathrm{a}}$, Aline Carneiro Viana ${ }^{\mathrm{b}}$, Mauro Fonseca ${ }^{\mathrm{a}}$ \\ ${ }^{a}$ Computer Department (DAINF), Graduate School of Electrical Engineering and \\ Computer Science (CPGEI), Federal University of Technology - Parana (UTFPR) \\ Av. Sete de Setembro, 3165. Rebouças. CEP 80230-901. Curitiba, Brazil. \\ \{criskochem, anelise, myriamdelg, maurof onseca\}@utfpr.edu.br \\ ${ }^{b}$ INRIA Saclay - Ile de France. 4, rue Jacques Monod, 91893 Orsay Cedex France. \\ Phone: +33 (0) 172925998 Fax: +33 (0) 174854249 \\ aline.viana@inria.fr
}

\begin{abstract}
This work proposes the Cultural Greedy Ant (CGrAnt) protocol to solve the problem of data delivery in opportunistic and intermittently connected networks referred to as Delay Tolerant Networks (DTNs). CGrAnt is a hybrid Swarm Intelligence-based forwarding protocol designed to address the dynamic and complex environment of DTNs. CGrAnt is based on: (1) Cultural Algorithms (CA) and Ant Colony Optimization (ACO) and (2) operational metrics that characterize the opportunistic social connectivity between wireless users. The most promising message forwarders are selected via a greedy transition rule based on local and global information captured from the DTN environment. Using simulations, we first analyze the influence of the ACO operators and CA knowledge on the CGrAnt performance. We then compare the performance of CGrAnt with the PROPHET and Epidemic protocols under varying networking parameters. The results show that CGrAnt achieves the highest delivery ratio (gains of $99.12 \%$ compared with PROPHET and $40.21 \%$ compared with Epidemic) and the lowest message replication (63.60\% lower than PROPHET and 60.84\% lower than Epidemic).
\end{abstract}

Keywords: cultural algorithms, ant colony, social analysis, forwarding protocol, intermittently connected networks.

\footnotetext{
${ }^{1}$ Corresponding Author: Ana Cristina Kochem Vendramin. DAINF/PPGCA/UTFPR. Av. Sete de Setembro, 3165. Rebouças. CEP 80230-901. Curitiba-PR, Brasil. Phone/Fax: +55(41)3310-4750/(41)3310-4646. criskochem@utfpr.edu.br
} 


\section{Introduction}

The pervasiveness of computing devices and the emergence of new applications and cloud services are factors emphasizing the increasing need for adaptive networking solutions. In most cases, this adaptation requires the design of interdisciplinary approaches as those inspired by nature, social structures, games, and control systems. The approach presented in this paper brings together solutions from different, yet complementary domains, i.e., networking, artificial intelligence, and complex networks, and is aimed at addressing the problem of efficient data delivery in intermittently connected networks.

As mobile devices become increasingly powerful in terms of communication capabilities, the appearance of opportunistic and intermittently connected networks referred to as Delay Tolerant Networks (DTNs) is becoming a reality (Khabbaz et al., 2012; Chaintreau et al., 2007; Tournoux et al., 2011). In such networks, contacts occur opportunistically in corporate environments such as conferences sites, urban areas, or university campuses. Understanding node mobility is of fundamental importance in DTNs when designing new communication protocols that consider opportunistic encounters among nodes. In fact, it is well known in the literature that the movement of nodes in such networks is not random and is a manifestation of their routine behavior and intentions (Gonzalez et al., 2008). Together with contact-based interactions among nodes, this movement generates a mobile social network. The analysis of such mobility patterns and the understanding of how mobile nodes interact (i.e., wirelessly encounter) play a critical role in the design of solutions for DTNs.

On the other hand, given that adaptation in nature is a permanent and continuous process, we note that the dynamic and complex environment of DTNs favors the application of Swarm Intelligence (SI) methods, including approaches based on Ant Colony Optimization (ACO) (Dorigo et al., 1996) and Cultural Algorithms (CAs) (Reynolds, 1994). In fact, the environment of opportunistic DTNs presents certain features in the mobility patterns of the network nodes that can be sufficiently explored by joining CA and ACO (e.g., knowledge stored in the belief space can guide the swarms through new or already constructed paths depending on the node behavior).

Motivated by those issues, this paper proposes the use of a Cultural 
Greedy Ant routing protocol, known as CGrAnt, to identify the most promising social-aware forwarders in DTNs, while profiting from SI-based paradigms. For this approach, the opportunistic and complex information (such as frequency and duration, centrality metrics, or mobility features) with respect to physical encounters between mobile nodes is gathered and favorable paths along which to forward each message are determined, while limiting data redundancy. Hence, the forwarding approach implemented by CGrAnt is adaptive and designed to match forwarding decisions to different mobility and operating conditions. Using a simulation environment, we evaluate the performance of CGrAnt under varying parameters, i.e., movement models, buffer sizes, message TTLs, simulation times, communication ranges, and transmission rates. The results confirm the satisfactory behavior of our routing protocol in key performance metrics, such as: message delivery ratio, message redundancy ratio, and message delivery delay.

The remainder of this paper is structured as follows. Section 2 provides an overview of the principles that drive our approach and its application environment. Section 3 describes the CGrAnt routing protocol in detail, and Section 4 presents the simulation environment. Sections 5 and 6 investigate how the proposed operational metrics and components affect the CGrAnt's performance. Section 7 compares the performance of CGrAnt with two known DTN forwarding protocols under varying networking parameters, and finally, Section 8 summarizes the concluding remarks and future directions.

\section{Rationale and Background}

This section begins with an overview of the addressed problem. The main innovations and contributions are further discussed, and the state-of-the-art of forwarding in DTN environment is described, with particular attention given to approaches based on SI.

\subsection{Problem overview}

In DTNs, a fully connected multi-hop path may not exist between a sender and a receiver due to either mobility issues or varying conditions of wireless communications, thus requiring the use of specific mechanisms to ensure robustness in the data communication among nodes. The information exchange must be performed in an opportunistic fashion through so-called carry-and-forward routing techniques (Cerf et al., 2007). The nodes may 
need to store messages from other nodes in their buffers for long periods of time and carry these messages until a forwarding opportunity arises (Cerf et al., 2007). Additionally, message replication may be necessary to increase the probability of successfully delivered messages. However, certain problems exist in a limited resource scenario: replications are undesirable because they compete with valid data messages in the paths toward a destination, and the storage of neighbors' data messages can be a problem due to limited buffer sizes.

The problem of routing in DTNs can thus, be modeled as a multimodal optimization problem attempting to find not just one solution but a set of solutions (i.e., multiple paths between two nodes). The finite set of possible solutions (i.e., paths formed by a sequence of nodes in which each node permutation generates a new solution) characterizes the routing in DTNs as a combinatorial problem. The problem can be also modeled as a dynamic state because the search space characteristics and the location and value of the solutions will change over time. The problem of routing in DTNs presents, therefore, a complex challenge, with several aspects still unexplored by most approaches described in the literature. Therefore, an updated consideration of the DTN dynamics is necessary and can be accomplished by periodically analyzing the neighbor information and selecting more than one path along which to forward each message while limiting message redundancy. The dynamicity and complex premises of DTNs characterize it as an environment favorable for the application of SI algorithms, including ACO and CA (Dorigo et al., 1996; Reynolds, 1994).

\subsection{CGrAnt in a Nutshell}

In view of the problem discussed in the previous session, we propose the CGrAnt protocol as a solution to the problem of identifying a set of good nodes along which to route each message in DTNs. To increase the reliability in such dynamic networks, choosing the best path for routing of messages should not be the main goal of a routing protocol. Indeed, it is equally important to maintain a diversity of paths and avoid convergence to only one or a few solutions.

CGrAnt can be defined as a hybrid SI system based on (1) CA and ACO and (2) operational metrics that characterize the opportunistic social connectivity between nodes. To adapt to the large topology variations encountered by a DTN and to reduce latency in message delivery, the following modifications are incorporated into CGrAnt that differentiate it from traditional 
SI-based protocols. (1) The SI control messages named Forward Ants (FAs), responsible for the path construction, are encapsulated into the data messages. (2) The number of FAs created and forwarded is dynamically defined according to the knowledge stored in the nodes. (3) CGrAnt adopts a greedy ACO transition rule that is similar to the deterministic transition rule proposed in the Ant Colony System (ACS) (Dorigo and Gambardella, 1997). Nevertheless, instead of using both probabilistic and deterministic rules as in ACS, CGrAnt uses only the greedy transition rule, which considers the heuristic function and/or pheromone concentration, to forward messages to the most promising node(s), and/or to exploit previously found good solutions. The search space exploration is still provided by the DTNs dynamics. (4) Instead of using time-based pheromone evaporation, CGrAnt performs an event-driven evaporation, which only occurs if a node detects that a new path toward a destination has been found. Thus, allowing redundant paths becomes more important than converging to the best path. (5) Because there is no central element in DTNs, the knowledge stored in the CA belief space is distributed among network nodes. (6) The information exchanged between the belief and population spaces always occurs in a distributed manner intermediated by the CGrAnt operational metrics. The ACO and CA modifications seem more adapted to intermittently connected networks such as DTNs, yet a subset may allow CGrAnt to operate in different dynamic scenarios.

\subsection{Related work}

We go through the related work in the area, discussing the most representative results on both DTN forwarding protocol and swarm intelligence methods.

\subsubsection{DTN Forwarding protocols}

The most common solutions in the literature take a controlled flooding approach. For instance, epidemic routing provides an optimal solution in terms of message delivery and latency, when no buffer constraint is present (Vahdat and Becker, 2000). In Epidemic routing, a node buffers a message and passes it on to all encountered nodes that have not received it before. No good message forwarders prediction is performed. To limit resource utilization, a hop-count field can be set in each message. Epidemic routing is simple and provides high reliability and adaptability, but it might generate too many 
redundant messages, wasting communication and battery resources. To reduce this overhead, the Spray and Wait approach (Spyropoulos et al., 2005) sprays messages over different contacts and then wait for these contacts to eventually deliver the message to the destination.

Predicted-based approaches try to reduce the message overhead by selecting a few good relays. In this context and more related to CGrAnt, several approaches estimate a delivery likelihood based on the frequency or similarities of meeting with contacts like PROPHET (Lindgren et al., 2003), Delegation Forwarding (Erramilli et al., 2008), and Spray and Focus (Spyropoulos et al., 2007). In particular, in PROPHET, vectors are exchanged that indicate the predictability of each node in delivering their messages. This predictability increases every time two nodes come into contact and reduces if they fail to meet frequently. When a node $A$ establishes a contact with a node $B$, a message will be sent to $B$ if its message delivery's prediction is higher as compared to $A$. The delivery predictability also has a transitive property. All these approaches, however, might be too conservative and lose good forwarding opportunities in environments with scarce connectivity. Most importantly, the majority of the approaches assume infinite buffers and bandwidth.

Other approaches study the effect of social networking on data forwarding. BubbleRap (Hui et al., 2008) and SimBet (Daly and Haahr, 2007) use information about social community structures and popularity within a community to choose good relays. (Zhang et al., 2012) introduce four social-aware data diffusion schemes based on the social relationship and data similarity of the contacts.

Differing from such protocols, CGrAnt conducts local and global searches and gathers relevant information from the DTN nodes. CGrAnt can thus analyze the utility of each node as a message forwarder and limit message replications.

\subsubsection{Swarm intelligence methods}

Though ACO has been extensively used in network environments, especially in MANETs (Liu and Feng, 2005; Rosati et al., 2008), routing in DTNs is challenging and few ACO protocols have been proposed. DAR (Rosati et al., 2008) does not consider local information from neighboring nodes and uses only the pheromone global information, which is not always available in DTNs. ABMF (La and Ranjan, 2009) only aims to estimate the extra capacity of each node as a message forwarder depending on its buffer dy- 
namics. ACRP (Zhang et al., 2010b) uses the Epidemic protocol to flood the network with control messages associated with Forward Ants (FAs) and Backward Ants (BAs). None of the mentioned approaches consider the following important aspects of sparse and opportunistic networks: (i) analyzing social metrics of nodes, including their degree and betweenness centralities to aid select message forwarders; (ii) preventing the loss of previously found good paths caused by pheromone evaporation processes that are periodically performed (i.e., based on time, as in ABMF and ACRP) or the overuse of those paths due to the absence of an evaporation process (as in DAR); and (iii) dynamically limiting the number of control and data messages forwarded in the network.

(Ma et al., 2008; Zhang et al., 2010a) use CA for performing routing in a static topology with service quality constraints. They, however, operate in a static environment and do not analyze the dynamics of the contacts in a social network to determine opportunities. Moreover, they search for a single optimal path with a set of constraints and use Situational and Normative knowledge only to increase the convergence speed. Finally, they use a single and centralized belief space.

Considering these issues, we proposed a first version of the SI-based routing protocol for DTNs that used only ACO (Vendramin et al., 2012b). Guided by pheromone concentration, heuristic functions, and social metrics, the Greedy Ant (GrAnt) protocol performed better than the well-known DTN routing protocols (Vendramin et al., 2012b).

CGrAnt encompasses CA and ACO metaheuristics and can be considered as an extension of our previous method (Vendramin et al., 2012b). CGrAnt improves the learning process and the gathering, during evolution, of highlevel information to be stored in the CA belief space.

\section{The CGrAnt Routing Protocol}

As previously mentioned, the CGrAnt routing protocol is based on CA and ACO meta-heuristics. CA is comprised of two spaces: (1) Belief Space, which represents the knowledge (i.e., set of information) gathered during the search for a set of paths and (2) Population Space, which is composed of individuals (i.e., ants) looking for solutions (i.e., forwarding paths) in an ACO framework.

In DTN, due to the lack of central element capable of storing and publishing all gathered information, the CGrAnt components are distributed among 


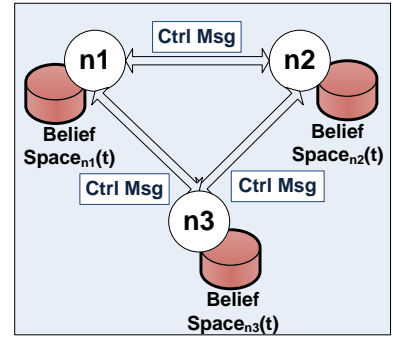

(a) Unsolicited

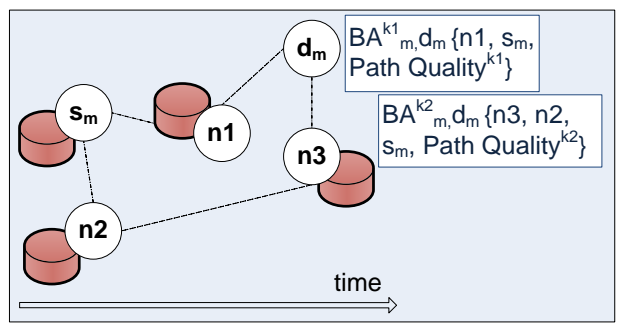

(c) On-Demand - Forward Part 2

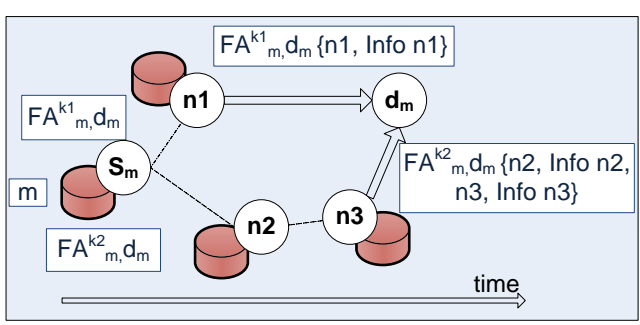

(b) On-Demand - Forward Part 1

Figure 1: Operation Modes of the CGrAnt Protocol.

different belief spaces, each stored in a network node; each node knows only a subset of the population space. The exchange of information between the belief and population spaces always occurs in a distributed manner.

CGrAnt operates in two modes: unsolicited and on-demand (Figure 1). In the unsolicited mode (Figure 1(a)), control messages (Ctrl Msg) are always exchanged among neighboring nodes to update the information stored in each belief space. If it is necessary to establish a data session between the source of a data message $m\left(s_{m}\right)$ and its destination $\left(d_{m}\right)$, CGrAnt switches to the on-demand mode (Figures 1(b)- 1(d)). In each node that contains a data message $m$ to be forwarded, one or more Forward Ants (FA) $k$, are forwarded toward $d_{m}$ along with $m$ via one or more neighboring nodes. During the path construction, an ant $k$ collects information (Info) on each node $n$ that composes the path toward $d_{m}$. The node $n$ can be either a node $i$ that contains a buffered message $m$ to be forwarded or a neighboring node $j$. A subset of this information is used by CGrAnt for the belief space update of each node. The other part is carried by the FA until it reaches $d_{m}$ (Figure $1(\mathrm{~b})$ ). In $d_{m}$, the quality of the constructed path is calculated based on the information gathered by the FA. A Backward Ant (BA) is sub- 
sequently created with the information obtained by the corresponding FA (Figure 1(c)), the FA is deleted, and the BA is sent back through the reverse path followed by the FA. In its path toward the source $\left(s_{m}\right)$ of the FA, the BA updates the ACO pheromone concentration operator $(\tau)$ in each link between the nodes that compose the reverse path (Figure 1(d)) according to the constructed path quality. At each visited node, its identification is removed from the BA's record. In subsequent message forwarding, the ACO operators (Pheromone Concentration and Heuristic Function) and the CA belief space (along with other CGrAnt components) dictate the routing decision in each node and infer the best forwarders for each message.

The next sections describe the CGrAnt routing protocol in detail. Sections 3.1 to 3.3 describe the components used by CGrAnt that influence the search for paths. Sections 3.4 and 3.5 describe the routing phases of CGrAnt, which determine the route(s) a message must follow to reach its destination.

\subsection{Metrics and Indicators}

The communication between the belief and population spaces is mediated by specific metrics and indicators. The metrics incorporated into CGrAnt are classified as basic (obtained directly from the population space or nodes) or composite (obtained from manipulating basic metrics). The basic metrics are classified into local (associated with each node and its neighboring nodes) and global (associated with complete paths constructed by ants) categories. Figure 2 illustrates the metrics and their relationships with the belief space stored in each node. The Situational and History knowledge influence the population space and the population space update the global metrics.

Table 1 provides a brief description of the metrics and variables used throughout this paper.

The Local Basic Metrics used by CGrAnt include the following:

- Frequency of Encounters $\left(F E_{n, d}\right)$ between a pair of nodes $n$ and $d$;

- Duration of an Encounter $\left(D E_{n, d}\right)$ between $n$ and $d$;

- Average Pause Time $\left(\overline{P T_{n}}\right)$ in the places visited by $n$;

- Average Movement Speed $\left(\overline{M S_{n}}\right)$ of a node $n$;

- Degree Centrality $\left(D C_{n}\right)$ of a node $n$ (Freeman, 1979). As $n$ encounters more nodes in the network and increments its degree centrality, it has more opportunities to choose the best message forwarders; 


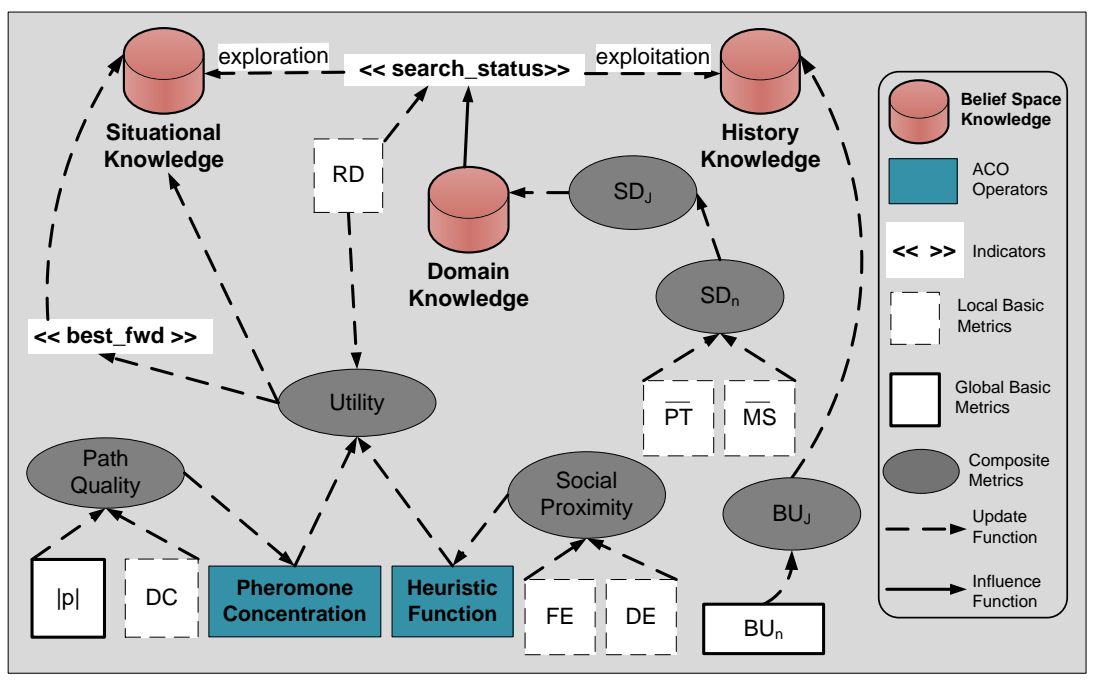

Figure 2: Belief Space of a Node.

- Relationship Degree $\left(R D_{i, m}\right)$ of a node $i$ with respect to each of its data messages $m . R D_{i, m}$ defines $i$ as the source $\left(s_{m}\right)$ or an intermediate node of $m$.

The Global Basic Metrics of CGrAnt include:

- Number of Hops $(|p|)$ in a complete path $p$. If there are few hops in a path, fewer resources are consumed, and less interference is generated;

- Betweenness Utility of a node $\left(B U_{n, d}\right) n$ relative to a destination node $d$. To obtain a high betweenness utility relative to $d$, a node $n$ must appear with a high frequency in paths between any source node and $d$. Each time a node $n$ receives a BA indicating that $n$ is a component of a complete path (global solution) to $d$, its betweenness utility is updated, $B U_{n, d}(t)=B U_{n, d}(t-1)+1$.

The Composite Metrics of CGrAnt include the following:

- Social Proximity $\left(S P_{n, d}\right)$ between $n$ and $d$ is directly associated with the ACO local operator Heuristic Function $(\eta)$ and is defined as $S P_{n, d}=F E_{n, d} \times D E_{n, d}$; 
- Path Quality $\left(Q_{p_{s_{m}, d_{m}}^{k}}\right)$, which measures the quality of a path $p$ constructed by an FA $k$ between nodes $s_{m}$ and $d_{m}$. It encompasses the number of hops $(|p|)$ and the average degree centrality of nodes $n\left(D C_{n}\right)$ that compose a path to $d_{m}$ :

$$
\mathrm{Q}_{p_{s_{m}, d_{m}}^{k}}(t)=\frac{\sum_{\forall n \in p_{s_{m}, d_{m}}^{k}} D C_{n}(t)}{|p|_{s_{m}, d_{m}}^{k}}+\frac{1}{|p|_{s_{m}, d_{m}}^{k}}
$$

The Path Quality metric is directly associated with the ACO global operator Pheromone Concentration $(\tau)$;

- Utility of a node $n$ in relation to $d\left(U_{n, d}\right)$, which describes how well $n$ can perform as a message forwarder to $d$. The $U_{n, d}$ is subsequently determined according to the basic metric $R D_{i, m}$ :

$$
U_{n, d}(t)= \begin{cases}\eta_{n, d}(t) & \text { if } R D_{i, m}=s_{m} \\ \eta_{n, d}(t)+\tau_{(i, y), d}(t) & \text { otherwise, }\end{cases}
$$

The $U_{n, d}(t)$ can thus consider only local or both local and global information. The local information $\eta_{n, d}(t)$ is the heuristic function of ACO measured by $S P_{n, d}$, and the global information $\tau_{(i, y), d}(t)$ is the pheromone concentration on each link $(i, y)$ belonging to a path to $d$, and $y$ is defined as:

$$
y= \begin{cases}d_{m} & \text { if } n=i \\ j & \text { if } n=j\end{cases}
$$

- Stagnation Degree $\left(S D_{n}\right)$ of a node $n$, which allows the identification of the most mobile nodes in a dynamic scenario (e.g., buses or vehicles) and consequently, adapts CGrAnt to heterogeneous networking encounters on the fly. For this, $S D_{n}$ considers the node average pause time $\left(\overline{P T}_{n}\right)$ and average movement speed $\left(\overline{M S}_{n}\right)$ :

$$
S D_{n}(t)=\left(\frac{\overline{P T}_{n}}{\overline{M S}_{n}}\right)
$$

- Stagnation Degree of the social network $\left(S D_{J}^{i}\right)$ of a node $i$, which is based on the $S D_{n}$ metric:

$$
S D_{J}^{i}(t)=\frac{1}{|J|} \sum_{j \in J} S D_{j}(t)
$$


where $J$ is the set of nodes encountered by node $i$;

- Betweenness Utility of the social network $\left(B U_{J, d}^{i}\right)$ of a node $i$ in relation to $d$, which considers the basic metric $B U_{n, d}$. The $B U_{J, d}^{i}$ is initialized differently depending on the origin of the BA that announces to $i$ that a complete path to $d$ has been constructed:

$$
B U_{J, d}^{i}(0)= \begin{cases}B U_{i, d}(t) & \text { if BA came from } d \\ B U_{j, d}(t) & \text { if BA came from } j\end{cases}
$$

The $B U_{J, d}^{i}$ metric is updated with $B U_{i, d}$ whenever $i$ receives a BA. When the BA comes from a neighboring node $j$ (not from $d$ ), $B U_{J, d}^{i}$ is also updated with $B U_{j, d}$ :

$$
B U_{J, d}^{i}(t)=\left\{\begin{array}{l}
Z \\
\text { if BA came from } d \\
\frac{1}{2}\left(Z+B U_{j, d}(t)\right) \\
\text { if BA came from } j,
\end{array}\right.
$$

where $Z=\frac{1}{2}\left(B U_{J, d}^{i}(t-1)+B U_{i, d}(t)\right)$.

More details on the metrics definitions are discussed in [23].

In addition to the basic and composite metrics, CGrAnt uses two indicators: (1) best_fwd $\mathbf{d}_{\mathbf{m}}$, which stores the current best forwarder for a specific message $m$ and (2) search_status, which decides whether FAs must explore or exploit the network while seeking solutions for the DTN forwarding problem.

\subsection{Population Space}

The population space is composed of FAs and BAs messages. The FAs look for sets of possible paths, i.e., one set $P_{m}$ for each pair $\left(s_{m}, d_{m}\right)$. Assuming a total of $M$ messages to be forwarded in the entire network, there will be several paths sets $\left\{P_{1}, \cdots, P_{m}, \cdots, P_{M}\right\}$ constructed simultaneously. Every paths set $P_{m}$ represents a group of solutions generated whenever a message $m$ originated in $s_{m}$ must be sent to $d_{m}$. Each complete path $p$ in $P_{m}$ composed of a sequence of nodes is established when ant $k$ reaches $d_{m}$ and can be defined as: 


$$
p_{s_{m}, d_{m}}^{k}=\left\{s_{m}, \cdots, n, \cdots, d_{m}\right\}, k=1, \cdots, K_{m} .
$$

For each node $i$ with a message $m$, each time a better forwarder $j$ for $m$ appears, a new FA $k$ is generated to begin its path construction, and a copy of $m$ is sent to $j$. The partially constructed path from $s_{m}$ to $j$ remains the same. However, from $j$, the FA $k$ is free to find $d_{m}$ passing through different nodes $n$ and can provide a different path into the population space. The $K_{m}$ defines the number of FAs generated to find solutions for $m$. In general, $\left|P_{m}\right| \ll K_{m}$ because a subset of ants cannot find $d_{m}$. The parameter $K_{m}$ is dynamically defined according to the belief space knowledge and message delivery success. Ant generation is thus auto-adaptive, as is the number of constructed paths, and both depend on the DTN dynamics.

\subsection{Belief Space}

In CGrAnt protocol, there is no element to centralize and share the gathered knowledge. The belief spaces are thus distributed over the network. Each belief space in a node encompasses three types of knowledge: Domain, History, and Situational, as detailed hereafter.

\subsubsection{Domain Knowledge}

introduced to support the analysis of local and specific DTN dynamics. Domain knowledge keeps CGrAnt updated on the relative local dynamics of each node $i$, which is evaluated in this paper based on the relationship between $S D_{i}$ and $S D_{J}^{i}$. The knowledge is distributed among nodes, $D o m_{i}$, for $i=1, \ldots, N$, where $N$ is the number of nodes available in the network and $D_{o m}$ assists CGrAnt in characterizing $i$ into three classes: a node with high, medium or low stagnation degree.

Based on this information and the specific heuristics of a DTN forwarding problem, the Domain knowledge can set the status of the path search (i.e., exploration or exploitation). The Acceptance and Update functions of the Domain knowledge occur more frequently than its Influence Function because they are called during the node encounters. The Influence Function acts only during the message forwarding phase.

The Acceptance Function accepts information on the local dynamic of each neighboring node $j$, only if $S D_{j} \in(0, \infty)$. After accepting a new solution, the Update Function is called. It considers the event window $W(t)$ 
containing a list of local events of $i$, such as an encounter between $i$ and a non-stationary node $j$.

The Update Function adds the pair $\left(S D_{j}(t), j\right)$ to the list and updates $S D_{J}^{i}$ according to Eq. 5.

The Influence Function evaluates the stagnation degree of $i$ with respect to its social network:

- High stagnation degree: A node $i$ is characterized as a highly stagnated node when the following relations apply: $S D_{i}>S D_{J}^{i}+V_{s}$ and $S D_{J}^{i} \in$ $(0, \infty)$. In this case, its improvement stagnation direction $d r_{i}$ is set to $d r_{i}=+1$

- Low stagnation degree: A node $i$ has a low stagnation degree when the following relations apply: $S D_{i}<S D_{J}^{i}-V_{i}$ and $S D_{J}^{i} \in(0, \infty)$ hold. In this case, $d r_{i}=-1$;

- Medium stagnation degree: A node $i$ is characterized as a medium stagnation node when the previously described relations do not apply. In this case, $d r_{i}=0$.

The $V_{i}$ and $V_{s}$ respectively define the decrement and increment in $S D_{J}^{i}$ used to define the range of nodes with medium stagnation degree.

Based on $d r_{i}$, the Influence Function changes the value of the search_status indicator, which aids in defining whether an FA in node $i$ must be sent to exploit or explore during the search for a solution of the DTN forwarding problem.

\subsubsection{History Knowledge}

introduced to adapt CGrAnt to changes in the network, thus increasing the ability to reflect the global network dynamics. This knowledge stores a history of important past events (in this case, the information that complete paths to $d$ have been found). Due to the lack of a central component, the complete paths cannot be stored in the History knowledge. The betweenness utility metric (representing the partial information of a complete solution) is subsequently used and may subsequently influence the path search. The History Knowledge is distributed among the network, $H i s_{i}$, for $i=1, \ldots, N$. In each node $i$, this knowledge is divided in a total of $D_{i}$ sub-knowledge: $H i s_{i}(t)=\left\{H i s_{i, 1}(t), H i s_{i, 2}(t), \ldots, H i s_{i, D_{i}}(t)\right\}$, where $D_{i} \leq N$ represents the number of destination nodes for which node $i$ originated or intermediated a path. 
The Acceptance and Update Functions of the History knowledge are called in the backward phase, and its Influence Function acts in the message forwarding phase.

The Acceptance Function is called after node $i$ receives a BA from a neighboring node $(j$ or $d)$, indicating that a complete path to $d$ has just been found. The betweenness utility of $i$ with respect to $d\left(B U_{i, d}\right)$ is always accepted to update the belief space of $i$. The betweenness utility of the neighboring node $j\left(B U_{j, d}\right)$ is also accepted to update the belief space of $i$ if and only if the BA did not come from $d$. After the acceptance phase, the Update Function is called to calculate $B U_{J, d}^{i}$.

The $B U_{j, d}$ and $B U_{J, d}^{i}$ dynamics are considered by the Influence Function when evaluating the improvement directions of each neighboring node $j$ as a candidate forwarder for message $m$ to $d$ : $d r_{j}=+1$, if $B U_{j, d}>B U_{J, d}^{i}$; $d r_{j}=-1$, if $B U_{j, d}<B U_{J, d}^{i}$; or $d r_{j}=0$, if $B U_{j, d}=B U_{J, d}^{i}$. Based on these directions and the search_status indicator, the history knowledge influences the message forwarding by deciding whether an FA should be sent through a previously found path and thus exploit the path search.

\subsubsection{Situational Knowledge}

introduced in CGrAnt to provide a memory of the best solutions, and to influence the search process for a set of paths through these solutions. Its memory is partial instead of complete and is represented by the best forwarder of each message. The Situational Knowledge is distributed among the network nodes: $S i t_{i}$, for $i=1, \ldots, N$. In each node $i$, the Situational knowledge is divided in a total of $M_{i}$ sub-knowledge:

$$
\operatorname{Sit}_{i}(t)=\left\{\operatorname{Sit}_{i, 1}(t), \ldots, \operatorname{Sit}_{i, m}(t), \ldots, \text { Sit }_{i, M_{i}}(t)\right\},
$$

where $M_{i}$ represents the number of data message $m$ stored in node i's buffer.

The Acceptance, Update, and Influence functions of the Situational knowledge are called during the message forwarding phase.

The Acceptance Function operates with the understanding that if a new neighboring node $j$ (partial solution) is found, it is accepted to update the belief space of $i$ only if $U_{j, d}>U_{\text {best_f } f d_{m}, d}$, where $U_{\text {best_f } f w d_{m}, d}$ is the current best forwarder utility for $m$ stored in the sub-knowledge $S_{i, m}$. The acceptance condition can be relaxed to accept nodes with the same utility of the best forwarder if the corresponding search_status exploration is true. Only one solution is accepted in each update. After accepting a new solution, the Update and Influence functions are called. The new solution thus replaces the previous solution (best $f w d_{m}=j$ ), and the new solution quality $\left(U_{j, d}\right)$ 
updates $U_{\text {best_f } f d_{m}, d}$ (i.e., $U_{\text {best_f } f w d_{m}, d}=U_{j, d}$ ). The Influence Function creates a new FA and forwards it along with $m$ to the new solution $j$. The Influence function thus dictates the future of each path built for a pair $s_{m}-d_{m}$ and the number of generated ants.

In the following sections, we describe in more detail the two phases that dictate the main functioning of the GrAnt routing protocol: (1) Message Forwarding or Path Search, which is represented by FAs looking for a set of paths and the updating of selected knowledge and metrics. In this phase occurs the data message forwarding; and (2) Backward, which is represented by BAs updating the knowledge and metrics stored in the nodes.

\subsection{Message Forwarding Phase}

The message forwarding phase in CGrAnt is initialized on-demand when a message $m$ stored in a node $i$ must be delivered to $d_{m}$, as described by Algorithm 1. The FAs are subsequently created, encapsulated into $m$, and sent toward $d_{m}$ via one or more neighboring nodes $j$.

During message forwarding, an FA at a node $i$ decides whether to forward $m$ to a new neighbor $j$ according to the influence of the three types of knowledge stored in its belief space: Domain, History, and Situational.

Because node $i$ is the first candidate solution for forwarding message $m$, its identification and utility initialize the Situational knowledge (lines 9 and 10). The decision on forwarding $m$ to $j$ may be to explore (i.e., it is not required that $j$ has previously participated in a path to $d_{m}$ ) or exploit (i.e., $j$ has previously participated in a path toward $d_{m}$ ). The decision is guided by the information on $i$ in terms of $R D_{i, m}$ and $S D_{i}$ stored in $D o m_{i}$. The status is initialized, enabling both exploitation and exploration of the path search space (lines 12 and 13). These conditions can change in two situations: (i) $i$ is an intermediate node with a medium stagnation degree (in this case, the exploration stops (line 16)), or (ii) $i$ is an intermediate node with a low stagnation degree (because $i$ is a highly mobile node, it does not forward $m$, thus, both exploration and exploitation are stopped (lines 19 and 20)).

Aside from Domain knowledge, the History and Situational knowledge also influence the CGrAnt message forwarding. The History knowledge influences the forwarding (line 33) when the decision is to exploit the solutions already found: $i$ forwards $m$ to a solution $j$ if node j's betweenness utility $\left(B U_{j, d}\right)$ is higher than the betweenness utility of node i's social network ( $B U_{J, d}^{i}$ stored in $H i s_{i, d}$, as observed in line 47 ). The Situational knowledge influences CGrAnt (line 44) when the decision is to explore the network or when 
better solutions appear. Node $i$ only forwards $m$ to a new solution $j$ if one of the following two conditions is satisfied: (i) The utility of $j\left(U_{j, d}\right)$ is higher than the utility of the best forwarder previously found for $m\left(U_{\text {best_f } f d_{m}, d}\right.$, which is stored in $S_{i, m}$, as observed in lines 53 and 54). This condition attempts to locate a new best forwarder for $m$ among the current neighboring nodes of $i$; (ii) At the beginning of the exploration (i.e., $m$ was not forwarded to any other node and best_f $w d_{m}=i$ and $U_{j, d}=U_{i, d}$, as in lines 26-27, 3638). The Situational and History knowledge are important contributions of CGrAnt because they dynamically control the number of created ants and the data message redundancy by setting each new best message forwarder or forwarding $m$ to already known good nodes, thus differing from the pure ACO algorithms proposed for DTNs.

After analyzing the utility of every current neighbor $j$ and inferring the best choice, CGrAnt sends $m$ to the designated forwarder (lines 33 and/or 44).

In addition to the data message forwarding, control messages are periodically and locally exchanged between $i$ and its neighboring nodes $j$ to update $\operatorname{Dom}_{i}$.

The search for new paths toward $d_{m}$ continues until $i$ performs one of the following actions: (1) encounters $d_{m},(2)$ becomes aware of the successful delivery of the corresponding data message to $d_{m}$, or (3) detects that the Time to Live (TTL) field of the data message has expired.

Throughout its path search, an FA carries the following information: the ID of $s_{m}$, the ID of $d_{m}$, the node IDs through which it passes (between $s_{m}$ and $\left.d_{m}\right)$, and the degree centrality of each visited node $j\left(D C_{j}\right)$. The individual qualities update the partial quality of the path under construction by the FA. When an FA (along with $m$ ) reaches $d_{m}$, the final quality of the constructed path $\left(Q_{p_{s m}^{k}, d_{m}}\right)$ is calculated as in Eq. 1. After calculating the quality of each new and complete path $p$, a new control message, the BA, is created from the information obtained by the FA, and the FA is deleted.

\subsection{Backward Phase}

During the backward phase, the BA returns to the node that originated the message $m$ through the reverse path selected by the FA. The concept of using a reverse path in DTNs is motivated by wireless social networks in which (i) individuals are often linked by a short chain of acquaintances, (ii) certain encounters show repetitive behavior, and (iii) nodes have routines that result in frequently visited locations and encounters. 
In the reverse path, receiving a BA sent from node $y$ to each neighboring node $i$ produces three effects: (1) increasing the $B U_{i, d}$ value by one; (2) initializing or updating the $B U_{J, d}^{i}$ value according to Eq. 6 and 7 , an effect that corresponds to the Update Function of the History Knowledge; and (3) updating the pheromone concentration toward $d$ according to:

$$
\tau_{(i, y), d}(t)=(1-\rho) \times \tau_{(i, y), d}(t-1)+Q_{p_{s_{m}, d_{m}}^{k}}(t),
$$

where $\tau_{(i, y), d}(t-1)$ is the pheromone on link $(i, y)$ that was last updated at time $(t-1)$. The evaporation process $(1-\rho)$ is necessary for the ants "to forget" the previous pheromone values deposited on a link to a specific $d$. This evaporation reduces the influence of the path search history. When the pheromone is updated, all concentrations that belong to $d$ are evaporated in $i$.

Even if the BA does not reach the node that originated the message (due to connection partitions), the message forwarding phase of the CGrAnt protocol is guided by a local path search provided by the heuristic function information. Differing from other protocols that use only global (pheromone concentration) or local (heuristic function) information, CGrAnt contains additional flexibility, because the decision is based on all available information (both ACO operators and knowledge stored in the CA belief space).

Additionally, the BA serves as an acknowledgment that $m$ has achieved $d_{m}$, allowing the nodes that still maintain $m$ to delete it and its associated variables. A node that encounters another node that has already received a BA for a given data message also deletes the corresponding message and its associated variables. When the source node receives it, the BA is deleted. Full paths are thus constructed for each destination using the information gathered by the ants during the path search phase.

\section{Evaluation Methodology}

This section describes the numerical analysis we conducted using the Opportunistic Network Environment (ONE) Simulator (Keränen et al., 2010) to investigate the benefits of the metrics and components incorporated into our proposal. Using ONE we can also assess both performance and accuracy of the CGrAnt protocol in simulation scenarios that consider two different movement models: Working Day (WD) (Ekman et al., 2008) and Points of Interest (PoI) (Keränen et al., 2010), both proposed by default in the ONE 
simulator. The ONE default parameters were kept unchanged (whenever possible) in order to confirm the results when compared with the others two evaluated protocols (Epidemic and Prophet). Moreover, using the default parameters, we intend to facilitate the dissemination of our proposal in the simulation platform.

The WD movement model represents an activity-based environment that simulates the daily lives (activities) of people who go to work in the morning, spend the day working, may go to a public place for leisure activities with friends at the end of day, and return to their houses at night. In WD, the total area $\left(10,000 \times 8,000 \mathrm{~m}^{2}\right)$ encompasses meeting points, buses, houses, offices, and roads. The area is divided into four regions denoted by $R_{A}$ to $R_{D}$. Eight groups of nodes, denoted by $A$ to $H$, are created to represent the node movements into specific regions. Groups $A$ to $D$ simulate only intraregion movements (e.g., group $A$ simulates the node movements into region $\left.R_{A}\right)$. Groups $E, F$, and $G$ simulate node movements between $R_{A}$ and other regions, and $H$ simulates movements among all regions. The assignment of nodes per group is as follows: $A$ has 258 nodes, $B$ has $119, C$ has $154, D$ has 154, $E$ has 102 (i.e., $E=A \cap B$ ), $F$ has 122 (i.e., $F=A \cap C$ ), $G$ has 122 (i.e., $G=A \cap D$ ), and $H$ has 70 (i.e., $H=A \cap B \cap C \cap D$ ).

The WD movement model represents an activity-based environment that simulates the daily lives (activities) of people who go to work in the morning, spend the day working, may go to a public place for leisure activities with friends at the end of day, and return to their houses at night. In WD, the total area $\left(10,000 \times 8,000 \mathrm{~m}^{2}\right)$ encompasses meeting points, buses, houses, offices, and roads. The area is divided into four regions denoted by $R_{A}$ to $R_{D}$. Eight groups of nodes, denoted by $A$ to $H$, are created to represent the node movements into specific regions. Groups $A$ to $D$ simulate only intraregion movements (e.g., group $A$ simulates the node movements into region $\left.R_{A}\right)$. Groups $E, F$, and $G$ simulate node movements between $R_{A}$ and other regions, and $H$ simulates movements among all regions. The assignment of nodes per group is as follows: $A$ has 258 nodes, $B$ has $119, C$ has $154, D$ has 154, $E$ has 102 (i.e., $E=A \cap B$ ), $F$ has 122 (i.e., $F=A \cap C$ ), $G$ has 122 (i.e., $G=A \cap D$ ), and $H$ has 70 (i.e., $H=A \cap B \cap C \cap D$ ).

In the PoI movement model, the total area $\left(8,800 \times 7,800 \mathrm{~m}^{2}\right)$ is divided into five points of interest that simulate several communities of people who eventually meet each other and exchange data. There are eight groups of nodes (W1, X1, Y1, Z1, W2, X2, Y2, and Z2), each with different destination selection probabilities. The POIs movement model is similar to the 
community model used by A. Lindgren et al.(Lindgren et al., 2003).

Groups W1, X1, Y1, and Z1 contain 30 nodes each. Groups W2, X2, Y2, and Z2 have five nodes. Every node has one home PoI that is more likely to be visited than the other PoIs; there is a high probability that nodes meet each other within their same home community and a low probability that they go to PoIs outside their home community. The nodes select a destination, move in this direction (with $\overline{M S} \in[0.5,1.5] \mathrm{m} / \mathrm{s}$ ), wait during a pause time $(\overline{P T})$ ranging from 100 to 200 seconds (for groups W1-Z1) or 4,000 to 5,000 seconds (for W2-Z2), and select the next destination, among other actions. Table 2 shows the settings and communication parameters applied in all experiments for the three protocols under comparison. Unless otherwise described, the parameters used in both scenarios are emphasized in Table 2.

In Section 5, we evaluate the CGrAnt performance with variations in the setting parameters. We analyze the CGrAnt performance in the PoI scenario in terms of the percentage of messages delivered to destinations. The setting parameters emphasized represent those that provide the best results for the CGrAnt protocol. Next, in Section 6, we investigate a subset of the CGrAnt components by evaluating the protocol performance for both scenarios (WD and PoI). Section 7 compares CGrAnt with the Epidemic and PROPHET protocols in both scenarios and considers different aspects of the communication network context. In all experiments, each message has a size of $500 \mathrm{~KB}$ representing its payload and includes an FA with 8 bytes representing its path quality. The BA size is 100 bytes on average (including the header, path quality, and path hops).

The results discussed in Sections 5, 6 and 7 are presented in terms of mean values and confidence intervals (at a 95\% confidence level) for 30 runs in each scenario. Due to the normality characteristics of the data under consideration, we apply the ANOVA (ANalysis Of VAriance) parametric statistical test together with its post hoc follow-up analysis over the independent groups considered. The ANOVA statistical test returns a p-value $>0.05$ indicating (with $95 \%$ of confidence) that there are no statistical differences among the groups and a p-value $<0.05$ if there is at least one pair of groups with a statistically significant difference. The intervals shown in the graphs for the post hoc analysis are computed in such a way that (to a close approximation) the two configurations compared are significantly different if their intervals are disjoint and are not significantly different if their intervals overlap. In our case, the delivery ratio interval associated with each group is represented by 
a horizontal line (with a circle representing its mean value). For each graph, we choose one group for emphasis (represented by a black horizontal line and delimited by two vertical dashed lines).

\section{Setting the Metrics of CGrAnt}

This section investigates how selected metrics and ACO operators can improve the communication among swarms in the population space and thus, assist in obtaining better solutions. Sections 5.1 and 5.2 analyze the influence of certain metrics associated with the ACO operators of CGrAnt. Section 5.3 analyzes the influence of selected metrics in characterizing the utility of each node (solution) as a message forwarder.

\subsection{Heuristic Function}

We first analyze different sources of information in the ACO local operator or Heuristic Function $\left(\eta_{n, d}(t)\right)$. Recall that a node $n$ is selected from a set of candidates to forward a message $m$ to its destination $d$. In this section, the CGrAnt performance is evaluated by considering each of the following metrics associated with $\eta_{n, d}(t):(1) S P_{n, d}$, (2) $D C_{n}$, (3) $B U_{n, d}$, and (4) $F B_{n}$, which represents the free space available in the buffer of a node $n$.

The experimental results show that when CGrAnt uses the $S P_{n, d}$ metric, it delivers more messages $(58.93 \pm 0.19 \%)$ than the $D C_{n}(47.21 \pm 0.17 \%)$, the $B U_{n, d}(53.92 \pm 0.17 \%)$, or the $F B_{n}(49.72 \pm 0.28 \%)$.

Figure 3 presents the post hoc analysis of ANOVA highlighting the $S P_{n, d}$ metric. The $S P_{n, d}$ metric guarantees the highest message delivery ratio. This associated to the fact that there are no overlaps among the intervals resulted from other metrics, lead us to conclude that $S P_{n, d}$ provides better performance than the other metrics.

The main reasons for the better performance of the $S P_{n, d}$ metric are listed as follows: (1) $S P_{n, d}$ indicates the proximity of $n$ relative to $d$ because it provides an estimation of the probability of future encounters between $n$ and $d$. Moreover, $S P_{n, d}$ contains information available along all of the search process. (2) $D C_{n}$ indicates the popularity of $n$ relative to all other nodes in the network instead of specific information for the candidate forwarder and $d$, as in $S P_{n, d}$. (3) $B U_{n, d}$ provides important information for the nodes that successfully intermediated a communication to $d$; however, it is only available for $n$ after a complete path has been found (which includes $n$ ), and $n$ has received the visit of a BA (as seen in Section 3.5). (4) In highly connected 


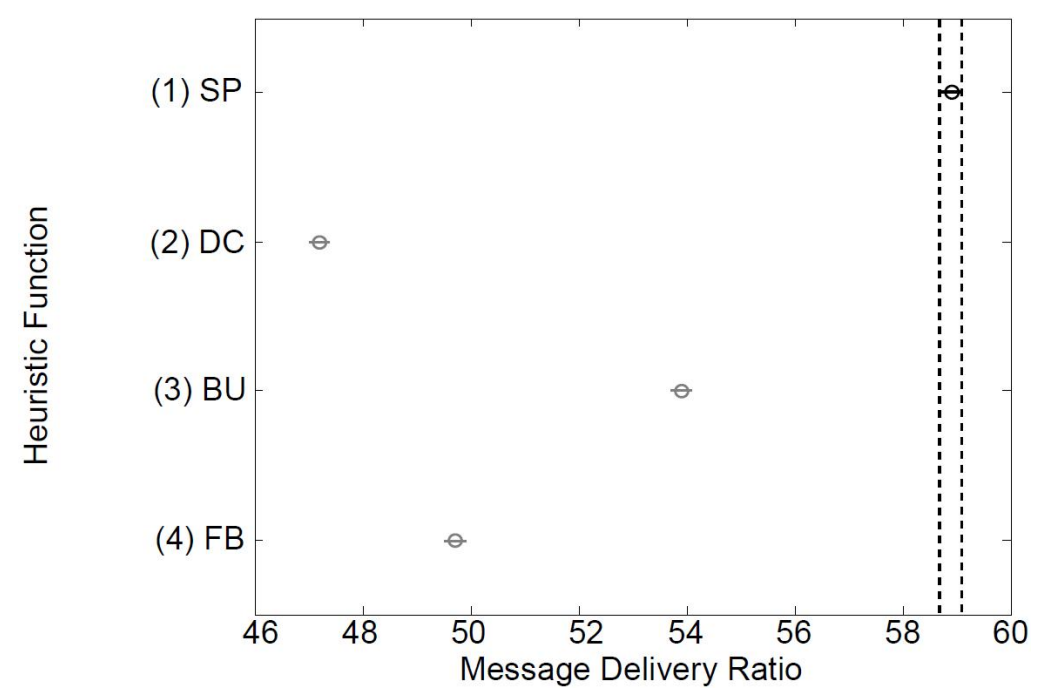

Figure 3: ANOVA results to Heuristic Function.

scenarios where the set of global solutions is always available and the same solution can be frequently selected, the percentage of available resources (e.g., the buffer) may be an important metric to consider; nevertheless, in an environment where the contacts are sparse (as in the DTNs), information on the social proximity between two nodes seems more important.

\subsection{Pheromone Concentration}

In this work, the pheromone concentration in the ACO global operator is associated with the quality of a complete path constructed by an ant $k$ between the $s_{m}$ and $d_{m}$ nodes (i.e., $\left.\tau_{(i, y), d}^{k}(t)=Q_{p_{s_{m}, d_{m}}^{k}}(t)\right)$. Next, after defining the Heuristic function, we evaluate which type of information have more influence on $Q_{p_{s_{m}, d_{m}}^{k}}(t)$. The CGrAnt performance is evaluated using different metrics to predict the path quality: (1) the average Degree Centrality $(D C)$ of nodes $n$ belonging to the path along with the reciprocal of the existing number of hops $(|p|)$ in the constructed path, i.e., as in Eq. 1; (2) only the second term of Eq. 1; (3) only the first term of Eq. 1; and (4) the average Betweenness Utility of node $n$ relative to $d$ (i.e., $Q_{p_{s_{m}, d_{m}}}(t)=\frac{\sum_{n} B U_{n, d}}{|p|}$ ).

The simulation results show that when using a composite metric encompassing $D C$ and $|p|$, CGrAnt delivers more messages $(58.93 \pm 0,19 \%)$ than when it uses only the basic metrics $|p|(51.11 \pm 0.22 \%), D C(51.10 \pm 0.21 \%)$, and $B U(49.89 \pm 0.22 \%)$. 
Figure 4 presents the post hoc analysis of ANOVA highlighting the composite metric $(D C$ and $|p|)$. As shown, the composite metric provides the best message delivery ratio. In addition, because there are no overlaps among the intervals of other metrics, we conclude that the composite metric provides better performance than the basic metrics. This result indicates that the node popularity is a good indicator of the node's ability to forward messages. This is particularly true in scenarios with intermittent connections, as in DTNs. Moreover, the importance of $|p|$ is due to the fact that the smaller is the path, the fewer are the network resources consumed and the less is the communication interference that occurs.

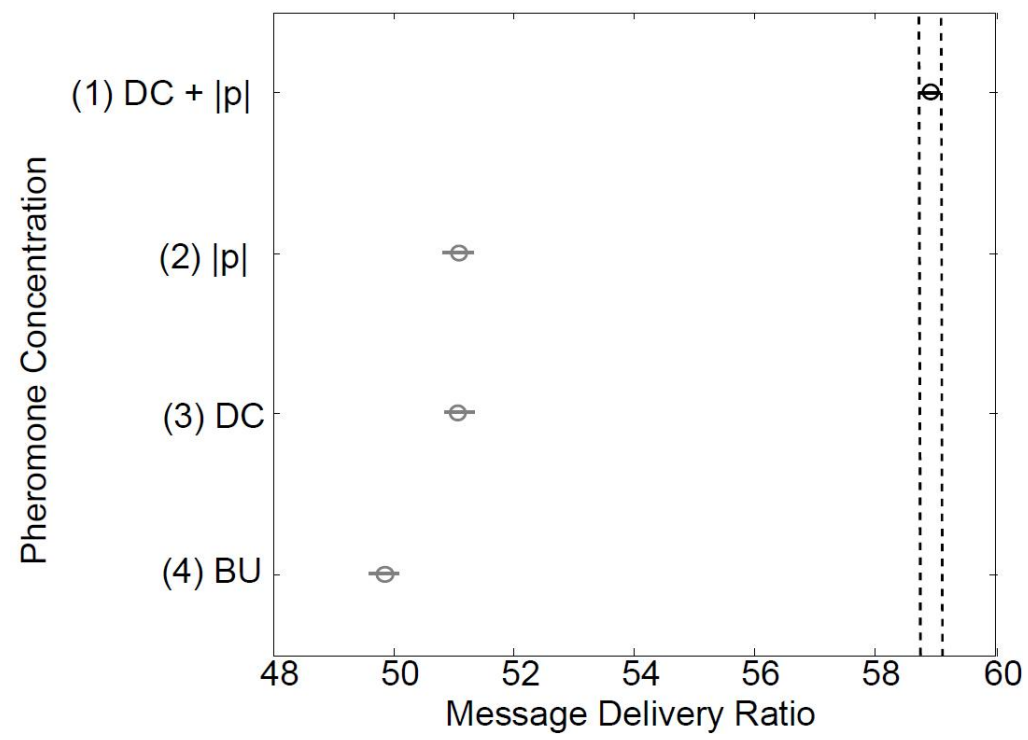

Figure 4: ANOVA results to Pheromone Concentration.

\subsection{Node Utility}

Finally, we analyze the utility $\left(U_{n, d}\right)$ of each node $n$ as a forwarder of a message $m$ to $d$. The CGrAnt performance is evaluated using four different metrics to describe the utility of $n$ related to a reference node $i$, which contains a message $m$ to be delivered to $d$. The investigated metrics are the following: (1) only local information represented by the Heuristic Function $\left(U_{n, d}=\eta_{n, d}(t)\right)$; (2) only global information represented by the Pheromone Concentration $\left(U_{n, d}=\tau_{(i, y), d}(t) ;(3)\right.$ the Heuristic Function and Pheromone Concentration $\left(U_{n, d}=\eta_{n, d}(t)+\tau_{(i, y), d}(t)\right)$; and (4) the Heuristic Function, 
Pheromone Concentration, and the Relationship Degree $\left(R D_{i, m}\right)$ metric (according to Eq. 2).

The results show that when both ACO operators (heuristic function and pheromone concentration) and the $R D_{i, m}$ metric are considered (composition 4 ), the CGrAnt protocol delivers more messages $(58.93 \pm 0.19 \%)$ compared with composition (1) in which it uses only the heuristic function (55.95 \pm $0.23 \%$ ), composition (2) in which it uses only the pheromone concentration $(49.29 \pm 0.18 \%)$, and composition (3) in which it uses the heuristic function and the pheromone concentration without the $R D_{i, m}$ metric $(58.02 \pm 0.20 \%)$.

Figure 5 presents the post hoc analysis of ANOVA highlighting the best composition (4). As depicted, there is no overlap among the intervals. This behavior verifies that the use of both local and global information (heuristic function and pheromone concentration) along with the $R D_{i, m}$ metric achieves higher performance.

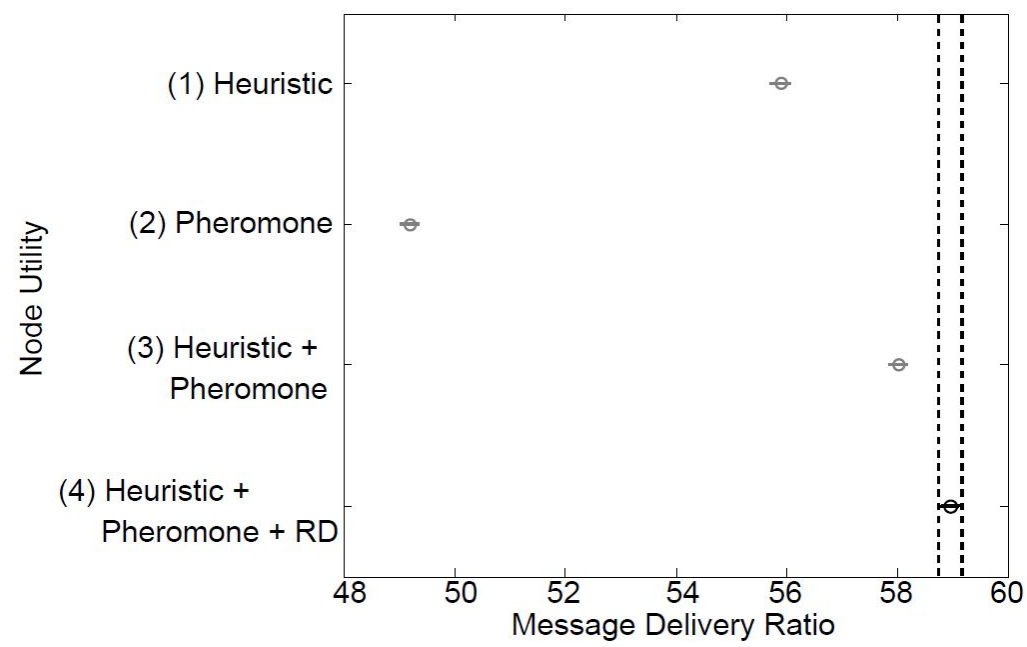

Figure 5: ANOVA results to Node's Utility Analysis.

\section{CGrAnt Component Analysis}

The influence of the CGrAnt's components on the message delivery ratio and message redundancy ratio are evaluated for the PoI and WD scenarios. The message redundancy ratio is expressed as Redundancy $=\left(B_{\text {transm }}-\right.$ $\left.B_{\text {delivery }}\right) / B_{\text {delivery }}$, in which $B_{\text {transm }}$ represents the number of bytes trans- 
mitted to nodes, and $B_{\text {delivery }}$, which is the number of bytes delivered to their destination.

Initially, an additive methodology is adopted in which components are added one by one to the previous protocol configuration until its final version (configuration 6) is reached (see Table 3). The first two configurations represent the CGrAnt protocol that considers only the ACO metaheuristic. The pheromone concentration slightly increases the delivery ratio and reduces the number of replicated messages. The influence of the new components incorporated into CGrAnt (i.e., CA's belief space) is analyzed for configurations 3 to 6 .

When comparing the results obtained from the pure ACO metaheuristic (configuration 2) with the final performance of CGrAnt (configuration 6), the following gains are achieved:

- in the POI scenario, the configuration 2 obtains $48.61 \%$ against $58.93 \%$ of message delivery in the configuration 6 . This represents a percentage increase of message delivery in $21.23 \%$;

- in the WD scenario, the configuration 2 obtains $54.97 \%$ against $63.19 \%$ of message delivery in the configuration 6 . This represents a percentage increase of message delivery in $14.95 \%$;

- in the POI scenario, the configuration 2 obtains $15.97 \%$ against $10.37 \%$ of message redundancy in the configuration 6 . This represents a percentage decrease of message redundancy in $35.07 \%$;

- in the WD scenario, the configuration 2 obtains $68.62 \%$ against $12.43 \%$ of message delivery in the configuration 6 . This represents a percentage decrease of message redundancy in $81.86 \%$.

The Situational knowledge (configuration 4) aims to dynamically restrict the number of FAs (and, consequently, the number of messages replicated) to only the most promising forwarders. The History knowledge (configuration 5) provides the exploitation of already known good solutions and consequently increases the message delivery ratio. The Domain knowledge 1 (configuration 3 ) privileges the exploration of the search space and the Domain knowledge 2 (configuration 6) favors the exploitation. According to the analysis of configurations 3 and 6, the Domain knowledge aims to increase the message delivery and reduce the redundancy ratio. 
GrAnt, our previous ACO-based protocol presented in (Vendramin et al., 2012b), performs better than CGrAnt in PoI (i.e., GrAnt provides $62.10 \pm$ $0.2 \%$ of message delivery and $7.05 \pm 0.1$ of message redundancy). However, for the WD scenario, CGrAnt outperforms GrAnt (the latter achieved $60.25 \pm$ $0.75 \%$ for delivery and $13.65 \pm 0.14$ for redundancy). CGrAnt presents better performance in WD rather than in POI scenario because of the Domain Knowledge. The Domain Knowledge considers the node mobility in order to determinate if a node is a good message forwarder and, consequently, if that node must exploit or explore the search space. In WD there are several mobility patterns, i.e., buses and vagabonds nodes with high mobility. Otherwise, in POI, the mobility of nodes is very similar and the information of the Domain knowledge is less relevant.

Additionally, CGrAnt has the advantage of modeling at a higher abstraction level that enables the elimination of any knowledge of the CA belief space in a simple way (e.g., the history knowledge can be eliminated when the environment is more connected and fewer messages need to be forwarded).

Table 4 represents the eliminatory analysis of the CA's belief space proposed by CGrAnt. The first configuration in Table 4 represents the CGrAnt protocol including all components. In analyzing this table we conclude the following:

- Domain Knowledge aims to increase the message delivery ratio and reduces the message replication. This is particularly true in the WD scenario in which the nodes generally have a stagnation degree $\left(S D_{n}\right)$ lower than the average stagnation of its social network $\left(S D_{J}\right)$, and consequently, the Domain knowledge has greater influence. Without this knowledge, the message delivery ratio is reduced by $1.77 \%$ (PoI) and $1.40 \%$ (WD) and the message redundancy ratio increased by $0.77 \%$ (PoI) and $92.27 \%(\mathrm{WD})$;

- Situational Knowledge aims to dynamically restrict the FAs to only good forwarders, and its influence on the message redundancy ratio is therefore greater. Without this knowledge influence, we observe an increase of $51.78 \%$ (PoI) and $248.51 \%$ (WD) in the message redundancy ratio and a reduction in the message delivery ratio of $9.15 \%$ (PoI). In the WD scenario we have an increase of message delivery ratio of 1.65\%;

- History Knowledge aims to enhance already known good solutions and thus increases the message delivery ratio. Without the influence of 
this knowledge, there is a reduction of $3.78 \%$ (PoI) and $3.4 \%$ (WD) in the message delivery ratio with a lowest cost incurred in the replication of messages $-33.85 \%$ (PoI) and $-12.55 \%$ (WD).

It may be noted that the use of the multiple knowledge incorporated in CGrAnt improves its final performance. As the use of multiple techniques in ensemble systems, the combination of a set of techniques on an suitable consensus function provides better performance than each individual technique.

\section{The CGrAnt Overall Performance}

This section investigates how CGrAnt performs as a forwarding protocol when compared with the Epidemic and PROPHET protocols under varying networking parameters. We performed 30 runs, and the reported results represent the mean and confidence intervals (at a $95 \%$ confidence level) values. To evaluate the reliability and the cost of the three protocols, we considered the following three performance metrics: (1) message delivery ratio, (2) message redundancy ratio, and (3) average message delivery delay.

\subsection{Analysis of Different Buffer Sizes}

Figure 6 depicts the performance of the three protocols with variation of the buffer sizes (from $4 \mathrm{MB}$ to $16 \mathrm{MB}$ ) for the PoI scenario. The dashed curves with empty points denotes the results for nodes that operate at a communication range of $10 \mathrm{~m}$ and a transmission rate of 2 Mbps (representing bluetooth devices). The solid curves with black points show the results for nodes that operate at a $100 \mathrm{~m}$ range and a transmission rate of 10 Mbps (representing WiFi devices). Figures 6(a) and 6(b) show that with the use of CGrAnt, more messages are delivered and less buffer space is devoted to message replications. For instance, for a buffer size of $8 \mathrm{MB}$ and a communication range of $100 \mathrm{~m}, \mathrm{CGrAnt}$ delivers $93.27 \pm 0.10 \%$ of messages (versus $66.52 \pm 0.16 \%$ for Epidemic and $46.84 \pm 0.15 \%$ for PROPHET) with a message replication of only $15.23 \pm 0.06 \%$ (38.90 $\pm 0.13 \%$ for Epidemic and $41.85 \pm 0.19 \%$ for PROPHET). Figure 6(c) shows that CGrAnt provides the lowest delivery delay when using a higher buffer size (i.e., $10 \mathrm{MB}$ to $16 \mathrm{MB}$ ).

Note that for buffer sizes lower than $8 \mathrm{MB}$, PROPHET presents better results in terms of delivery delay. This is due to its lowest Message Delivery ratio, almost $-50 \%$ than CGrAnt, only short route with short delivery delay is used. 


\subsection{Analysis of Different Message TTLs}

Figure 7 shows the performance of the CGrAnt, Epidemic, and PROPHET protocols with variation of the message TTL (Time-To-Live, i.e., how long the message lives in the network in minutes) for the PoI scenario. Figures 7(a) and $7(\mathrm{~b})$ show that CGrAnt provides the best results in terms of message delivery and redundancy ratios for all message TTLs and both communication ranges. For instance, with a $10 \mathrm{~m}$ range and a TTL of 2,100 minutes, CGrAnt delivers $61.09 \pm 0.23 \%$ of messages (versus $35.39 \pm 0.17 \%$ for Epidemic, $30.50 \pm 0.14 \%$ for PROPHET), with a message redundancy of only $10.21 \pm 0.05 \%$ (30.47 $\pm 0.18 \%$ for Epidemic, $32.82 \pm 0.17 \%$ for PROPHET). These results show that a node with an efficient routing protocol such as CGrAnt, with guidance from the CA knowledge and the ACO operators is able to efficiently manage message forwarding and dynamically limit message redundancy. Figure $7(\mathrm{c})$ shows that PROPHET provided the best results in terms of delivery delay; this is the only metric for which CGrAnt cannot provide the best results, a lack that is justified by its lowest Message Delivery ratio, almost $-40 \%$ than CGrAnt, only short route with short delivery delay is used.

The performance of the three DTN protocols with variations in the buffer sizes and message TTLs in the WD scenario is presented in (Vendramin et al., 2012a). The results in (Vendramin et al., 2012a) show that CGrAnt achieves a higher message delivery ratio and a lower redundancy ratio than those of Epidemic and PROPHET.

\subsection{Analysis of Different Simulation Times}

We also perform an experiment to evaluate the number of messages delivered by the three protocols along the simulation time in the PoI (4MB of buffer size and message TTL of 600) and the WD scenarios (10MB of buffer size and message TTL of 1,800$)$, both with a communication range of $10 \mathrm{~m}$. The aim in this section is to demonstrate that a better delivery ratio can be achieved as the time increases.

Figure 8 shows the message delivery ratio obtained by the three protocols over time in the PoI and WD scenarios with a $10 \mathrm{~m}$ range. In CGrAnt, the performance gain is greater mainly in the WD scenario. When the simulation time is increased from 400,000 to 2,800,000 seconds, CGrAnt delivers slightly better performance via an increase of $7.37 \%$ (PoI) and $17.45 \%$ (WD) in the message delivery ratio. This gain is justified by the fact that the more is the gathered information by CGrAnt over time, the better are the choices it can 
make concerning message forwarding candidates. In contrast, PROPHET and Epidemic did not exhibit any significant variation of performance when the simulation time was increased.

\subsection{Analysis of Operation Costs}

Another important consideration in protocol performance is the cost of initializing/updating and storing the state of the network and nodes. This cost covers the amount of the following types of information: (1) locally exchanged between every two nodes $i$ and $j$ during any contact opportunity, and (2) locally stored in each node $i$ of the network. For this analysis, we considered the PoI scenario with the emphasized parameters presented in Table 2.

For the storage cost in terms of the total number of bytes exchanged between nodes $i$ and $j$ during every contact opportunity, PROPHET displays a higher cost due to the exchange of its delivery predictability list. Due to the transitive property of PROPHET, the number of records in the predictability list of a node rapidly reaches the total number of network nodes (139, excluding itself). Thus, the number of bytes exchanged in both directions of the contact is 2,224 bytes $(139 \times 16$ bytes $)$, as it is shown in Table 5 . For the CGrAnt protocol, during the message forwarding phase, 16 bytes are sent by $i$ to its neighboring node $j$, identifying a data message $m$ to be sent and the stagnation degree of $i$. At the same time, 29 bytes are sent by $j$ to $i$ representing node $j$ on how well it can perform as a forwarder for $m$ : including its stagnation degree, its degree centrality, its social proximity with the destination $d$ of the message $m$ stored in node $i$, its betweenness utility relative to $d$, and an indication (true or false) that it knows that $m$ was already received by $d$.

For the cost in terms of the total number of bytes stored in each node $i$, because Epidemic relies on the message replications to eventually deliver its messages, its storage cost is null (i.e., it is a stateless protocol). Although CGrAnt generates a higher storage cost compared with PROPHET and Epidemic, in the worst case, that cost $(11.28 \mathrm{~KB})$ represents only $0.28 \%$ of the total capacity of a node buffer, if considering a limiting buffer size of $4 \mathrm{MB}$ per node.

Finally, we investigate the operational cost provided by CGrAnt when considering only its control messages related to the ACO ants (FAs and BAs) in both PoI (buffer of $4 \mathrm{MB}$ and message TTL of 600 minutes) and WD (buffer of $10 \mathrm{MB}$ and message TTL of 1,800 minutes) scenarios. In the PoI 
scenario, the control bytes corresponding to the FAs and BAs represent only $0.0131 \pm 0,0009 \%$ (communication range of $10 \mathrm{~m}$ ) and $0.0186 \pm 0,0002 \%$ (100 $\mathrm{m}$ range) of the total bytes generated by CGrAnt (counting the bytes related to data messages, FAs, and BAs). In the WD scenario, the FAs and BAs bytes represent, respectively, $0.0194 \pm 0,0003 \%(10 \mathrm{~m})$ and $0.079 \pm 0,0022 \%$ $(100 \mathrm{~m})$ of the total bytes generated. Nevertheless, even if accounting for the extra cost of these control bytes (for the FAs and BAs) in the total amount (in bytes) of replicated messages in the network, CGrAnt propagates fewer bytes in the network compared with Epidemic and PROPHET due to the high number of data messages replicated by the latter protocols. When compared with Epidemic, CGrAnt provides a reduction of $39.99 \pm 0,16 \%$ (in PoI with a $10 \mathrm{~m}$ range), $59.60 \pm 0,11 \%$ (PoI with a $100 \mathrm{~m}$ range), $83.60 \pm 0,19 \%$ (WD with a $10 \mathrm{~m}$ range), and $89.49 \pm 0,26 \%$ (WD with a $100 \mathrm{~m}$ range) in the total number of bytes generated in the network. Similarly, when compared with PROPHET, these reductions are 34.86 \pm , 18\% (PoI with 10 $\mathrm{m}$ ), $43.37 \pm 0,15 \%$ (PoI with $100 \mathrm{~m}$ ), $74.59 \pm 0,34 \%$ (WD with $10 \mathrm{~m}$ ), and $78.57 \pm 0,56 \%$ (WD with $100 \mathrm{~m}$ ) in total bytes generated in the network. Therefore, we conclude that with the smallest generated overhead, CGrAnt is able to choose the best message forwarders and reduce the total number of data bytes replicated in the network.

It is important to highlight that the algorithmic complexity for the CGrAnt protocol is linear $[\mathrm{O}(\mathrm{n})]$ in the number of network nodes, as shown in Algorithm 1.

\section{Conclusions}

The importance of inferring the social behavior of nodes to efficiently deliver data in mobile and intermittently connected networks has motivated the development of the hybrid swarm intelligence-based CGrAnt protocol. Using a greedy version of ACO and CA, CGrAnt characterizes the utility of each node as a message forwarder by considering a set of social-aware metrics. We performed a set of experiments to analyze the influence of selected metrics associated with the ACO operators and the use of different metrics to characterize the utility of each node as a message forwarder. Once the group of metrics was set, we analyzed the influence of the ACO operators and CA knowledge on the CGrAnt performance. Finally, we compared the performance of CGrAnt with the Epidemic and PROPHET protocols under varying networking parameters. The simulation results showed that CGrAnt outper- 
formed PROPHET and Epidemic forwarding protocols in terms of message delivery (gains of $99.12 \%$ compared with PROPHET and $40.21 \%$ compared with Epidemic) and message replication (63.60\% lower than PROPHET and $60.84 \%$ lower than Epidemic). In addition, despite a higher storage cost compared to PROPHET and Epidemic (11.28 KB in the worst case), CGrAnt propagates fewer bytes in the network due to the high number of data messages replicated by the latter protocols (a reduction of $43.37 \%$ when compared to PROPHET and $59.60 \%$ when compared to Epidemic). In future work, we intend to study in more details the adaptive capabilities of CGrAnt, when operating in a scenario with varying mobility conditions, i.e., from an almost static to a completely mobile and disconnected networking environment. For this work, we will investigate the self-adaptation of social-aware metrics, which can be combined with and applied to each CGrAnt component. Finally, the comparison of CGrAnt performance with other related social-based forwarding protocols as well as the use of real data sets is such analysis will be also let for future works.

\section{Curriculum Vitae}

Ana Cristina B. Kochem Vendramin received her graduate degree in Computer Science from Paranaense University in 2000 and Ph.D. degree in Computer Engineering from UTFPR in 2012. Currently, she is a Professor in DAINF Department at UTFPR. Her research interests include distributed systems, swarm intelligence, routing in cellular and mobile ad hoc networks.

Anelise Munaretto received her Dipl.-Ing. degree in Computer Engineering from PUPCPR in 1994 and Ph.D. degree in Computer Networks from the University of Paris VI in 2004. Currently, she is Associate Professor at UTFPR. Her research interests include routing and quality of service optimization in wireless networks.

Myriam Regattieri Delgado received her graduate degree at Electrical Engineering from UFG in 1990 and Ph.D. degree in Computer Engineering from UNICAMP in 2002. Currently, she is associate professor at UTFPR. Her research interests include natural computing, swarm intelligence applied in computer networks.

Aline Carneiro Viana is a Researcher at INRIA Saclay. She got her Ph.D. in Computer Science from the University Pierre et Marie Curie in 2005. After holding a postdoctoral position at IRISA/INRIA Rennes, she joined 
INRIA Saclay in 2006. Her research is primarily in success data delivery in wireless self-organized and adaptive networks.

Mauro Fonseca received the B.Sc Degree in Computers Engineering from the PUCPR in 1994 the Ph.D. degree in Computer Networks from University Pierre et Marie Curie in 2003. He is currently an associate professor at UTFPR. His research interests include wireless networks and network performance.

\section{Acknowledgements}

This work is partially supported (1) by the Brazilian National Research Council (CNPq), under research grants 309571/2014-6 to A. Munaretto and 309197/2014-7 to M.R. Delgado, and 479159/2013-0 to A. Munaretto and (2) by the STIC AmSud UCOOL project.

\section{References}

Cerf, V., Burleigh, S., Hooke, A., Torgerson, L., Durst, R., Scott, K., Fall, K., Weiss, H., 2007. Delay-Tolerant Network Architecture. IETF RFC-4839, DTN Research Group. URL http://www.ietf.org/rfc/rfc4838.txt (Last access: March, 2015)

Chaintreau, A., Hui, P., Scott, J., Gass, R., Crowcroft, J., Diot, C., 2007. An improved ant-based algorithm for the degree-constrained minimum spanning tree problem. IEEE Trans. Mobile Computing 6 (6), 606-620.

Daly, E. M., Haahr, M., 2007. Social network analysis for routing in disconnected delay-tolerant MANETs. In: Proceedings of the 8th ACM International Symposium on Mobile Ad Hoc Networking and Computing. Montreal, Canada, pp. 32-40.

Dorigo, M., Gambardella, L. M., 1997. Ant colony system: A cooperative learning approach to the traveling salesman problem. IEEE Trans. Evol. Comput. 1 (1), 53-66.

Dorigo, M., Maniezzo, V., Colorni, A., 1996. The ant system: Optimization by a colony of cooperating agents. IEEE Trans. Syst., Man, Cybern. B $26(1), 29-41$. 
Ekman, F., Keränen, A., Karvo, J., Ott, J., 2008. Working day movement model. In: Proceedings of 1st ACM/SIGMOBILE Workshop on Mobility Models. Hong Kong, China, pp. 33-40.

Erramilli, V., Crovella, M., Chaintreau, A., Diot, C., 2008. Delegation forwarding. In: Proceeding of Mobihoc 2008. Hong Kong, China, pp. 251-259.

Freeman, L. C., 1979. Centrality in social networks: Conceptual clarification. Social Networks 1 (3), 215-239.

Gonzalez, M. C., Hidalgo, C. A., Barabasi, A.-L., 2008. Understanding individual human mobility patterns. Nature 453 (5), 779-782.

Hui, P., Crowcroft, J., Yoneki, E., 2008. BUBBLE Rap: Social-based forwarding in delay tolerant networks. In: Proceedings of Mobihoc 2008. Hong Kong, China, pp. 241-250.

Keränen, A., Kärkkäinen, T., Ott, J., 2010. Simulating mobility and DTNs with the ONE. J. of Communications 5 (2), 92-105.

Khabbaz, M. J., Assi, C. M., Fawaz, W. F., 2012. Disruption-tolerant networking: A comprehensive survey on recent developments and persisting challenges. IEEE Commun. Surveys Tuts. 14 (2), 607-640.

La, R. J., Ranjan, P., 2009. Ant-based adaptive message forwarding scheme for challenged networks with sparse connectivity. In: Proceedings of the 28th IEEE Conference on Military Communications (MILCOM). Boston, USA, pp. 1-7.

Lindgren, A., Doria, A., Schelén, O., 2003. Probabilistic routing in intermittently connected networks. SIGMOBILE Mob. Comput. Commun. Rev. 7 (3), 19-20.

Liu, L., Feng, G., 2005. Swarm intelligence based node-disjoint multi-path routing protocol for mobile ad hoc networks. In: Proceedings of the Fifth International Conference on Information, Communications and Signal Processing (ICICS). Bangkok, Thailand, pp. 598-602.

Ma, J., p. Zhang, J., Yang, J., l. Cheng, L., 2008. Research on cultural algorithm for solving routing problem of mobile agents. Journal of Chine Univ. of Posts and Telecom. 15 (4), 121-125. 
Reynolds, R. G., 1994. An introduction to cultural algorithm. In: Proceedings of the 3rd Annual Conference on Evolutionary Programming. Vol. 41. San Diego, USA, pp. 131-139.

Rosati, L., Berioli, M., Reali, G., 2008. On ant routing algorithms in ad hoc networks with critical connectivity. Ad Hoc Networks 6 (6), 827-859.

Spyropoulos, T., Psounis, K., Raghavendra, C. S., 2005. Spray and wait: An efficient routing scheme for intermittently connected mobile networks. In: Proceedings of the 2005 ACM SIGCOMM Workshop on Delay-tolerant Networking. WDTN '05. Philadelphia, USA, pp. 252-259.

Spyropoulos, T., Psounis, K., Raghavendra, C. S., 2007. Spray and focus: Efficient mobility-assisted routing for heterogeneous and correlated mobility. In: Proceedings of Fifth Annual IEEE International Conference on Pervasive Computing and Communications Workshops. IEEE PerCom Workshops '07. White Plains, NY, USA, pp. 79-85.

Tournoux, P.-U., Leguay, J., Benbadis, F., Whitbeck, J., C., V., de Amorim, M. D., 2011. Density-aware routing in highly dynamic dtns: The rollernet case. IEEE Trans. Mobile Computing 10 (12), 1755-1768.

Vahdat, A., Becker, D., 2000. Epidemic routing for partially connected ad hoc networks. Tech. rep., duke University. Technical Report-CS-2000-06.

URL http://www.cs.duke.edu/ vahdat/ps/epidemic.pdf (Last access: April, 2015)

Vendramin, A. C. B. K., Munaretto, A., Delgado, M., Viana, A. C., $2012 a$. CGrAnt: A swarm intelligence-based routing protocol for delay tolerant networks. In: Proceedings of the 14th Annual Conference on Genetic and Evolutionary Computation. GECCO '12. Philadelphia, USA, pp. 33-40.

Vendramin, A. C. B. K., Munaretto, A., Delgado, M., Viana, A. C., 2012b. GrAnt: Inferring best forwarders from complex networks' dynamics through a greedy ant colony optimization. Computer Networks 56 (3), 997-1015.

Zhang, M.-W., Sun, X.-M., Lv, X.-Y., 2010a. A QoS routing algorithm based on culture-ant colony algorithm. In: Proceedings of the International Conference on Computer Application and System Modeling. Taiyuan, China, pp. 198-201. 
Zhang, P., Wang, H., Xia, C., Lv, L., Liu, X., 2010b. ACRP: Ant-colonybased routing protocol for DTMNs. In: Proceedings of the International Conference on Educational and Information Technology. Chongqing, China, pp. 272-276.

Zhang, Y., Gao, W., Cao, G., Porta, T. L., Krishnamachari, B., Iyengar, A., 2012. Social-aware data diffusion in delay tolerant manets. Handbook of Optimization in Complex Networks. Springer Optimization and Its Applications 58, 457-481. 
Table 1: Summary of the metrics and variables used to describe the network and CGrAnt

\begin{tabular}{|c|c|}
\hline \multicolumn{2}{|r|}{ Network Variables } \\
\hline$m$ & A specific data message \\
\hline$s$ & A general source node $\left(s_{m}:\right.$ refers to the source of $\left.m\right)$ \\
\hline$d$ & A general destination node $\left(d_{m}:\right.$ the destination node of $\left.m\right)$ \\
\hline$i$ & Node with a data message to be forwarded \\
\hline$j$ & Neighboring node of $i$ \\
\hline$n$ & A generic node, $i$ or $j$ \\
\hline$y$ & A generic node, $j$ or $d$ in a link toward $d$ \\
\hline$N$ & The total number of nodes available in the network \\
\hline$M$ & The total number of data messages to be forwarded \\
\hline$J$ & Set of nodes $j$ encountered by the node $i$ (social network of $i$ ) \\
\hline$D_{i}$ & $\begin{array}{l}\text { The total number of destination nodes for which } i \\
\text { originated or intermediated a path }\end{array}$ \\
\hline \multicolumn{2}{|r|}{$\begin{array}{c}\text { CGrAnt Variables } \\
\end{array}$} \\
\hline$F A / B A$ & A general Forward/Backward Ant \\
\hline$k$ & A specific FA/BA \\
\hline$K_{m}$ & The total number of FAs generated for a message $m$ \\
\hline$p$ & A complete path with a total of $|p|$ hops \\
\hline$P_{m}$ & Group of paths $p$ constructed by ants for a message $m$ \\
\hline$W$ & Event window \\
\hline$d r_{n}$ & Improvement direction of a node $n$ \\
\hline$V_{i} / V_{s}$ & $\begin{array}{l}\text { Inferior/Superior limits which define the } \\
\text { range of medium stagnation degree }\end{array}$ \\
\hline \multicolumn{2}{|r|}{ CGrAnt Local Metrics } \\
\hline$F E_{n, d}$ & Frequency of Encounters between $n$ and $d$ \\
\hline$D E_{n, d}$ & Duration of an Encounter between $n$ and $d$ \\
\hline$\overline{P T}_{n}$ & Average Pause Time in the places visited by a node $n$ \\
\hline$\overline{M S} S_{n}$ & Average Movement Speed of a node $n$ \\
\hline$D C_{n}$ & Degree Centrality of a node $n$ \\
\hline$R D_{i, m}$ & $\begin{array}{l}\text { Relationship Degree of a node } i \text { with respect to a } \\
\text { specific buffered data message } m\end{array}$ \\
\hline \multicolumn{2}{|r|}{ CGrAnt Global Metrics } \\
\hline$|p|$ & Number of hops in a complete path $p$ \\
\hline$B U_{n, d}$ & Betweenness Utility of a node $n$ in relation to $d$ \\
\hline \multicolumn{2}{|r|}{ CGrAnt Composite Metrics } \\
\hline$B U_{J, d}^{i}$ & $\begin{array}{l}\text { Betweenness Utility of the social network of } i \\
\text { in relation to a destination } d\end{array}$ \\
\hline$S P_{n, d}$ & Social Proximity between nodes $n$ and $d$ \\
\hline$U_{n, d}$ & Utility of a node $n$ as a message forwarder to $d$ \\
\hline$\eta_{n, d}$ & Heuristic Function measured by $S P_{n, d}$ \\
\hline$\tau_{(i, y), d}$ & $\begin{array}{l}\text { Pheromone concentration on each link }(i, y) \\
\text { belonging to a path to node } d\end{array}$ \\
\hline$S D_{n}$ & Stagnation Degree of a node $n$ \\
\hline$S D_{J}^{i}$ & Stagnation Degree of the social network of node $i$ \\
\hline$Q_{p_{s m, d m}^{k}}$ & Quality of a path $p$ (from $s_{m}$ to $\left.d_{m}\right)$ constructed by FA $k$ \\
\hline \multicolumn{2}{|r|}{ CGrAnt Knowledge } \\
\hline $\operatorname{Dom}_{i}$ & Domain Knowledge of node $i$ \\
\hline$H_{i s}{ }_{i, d}$ & History Knowledge of $i$ with respect to $d$ \\
\hline Sit $_{i, m}$ & Situational Knowledge of $i$ with respect to $m$ \\
\hline
\end{tabular}




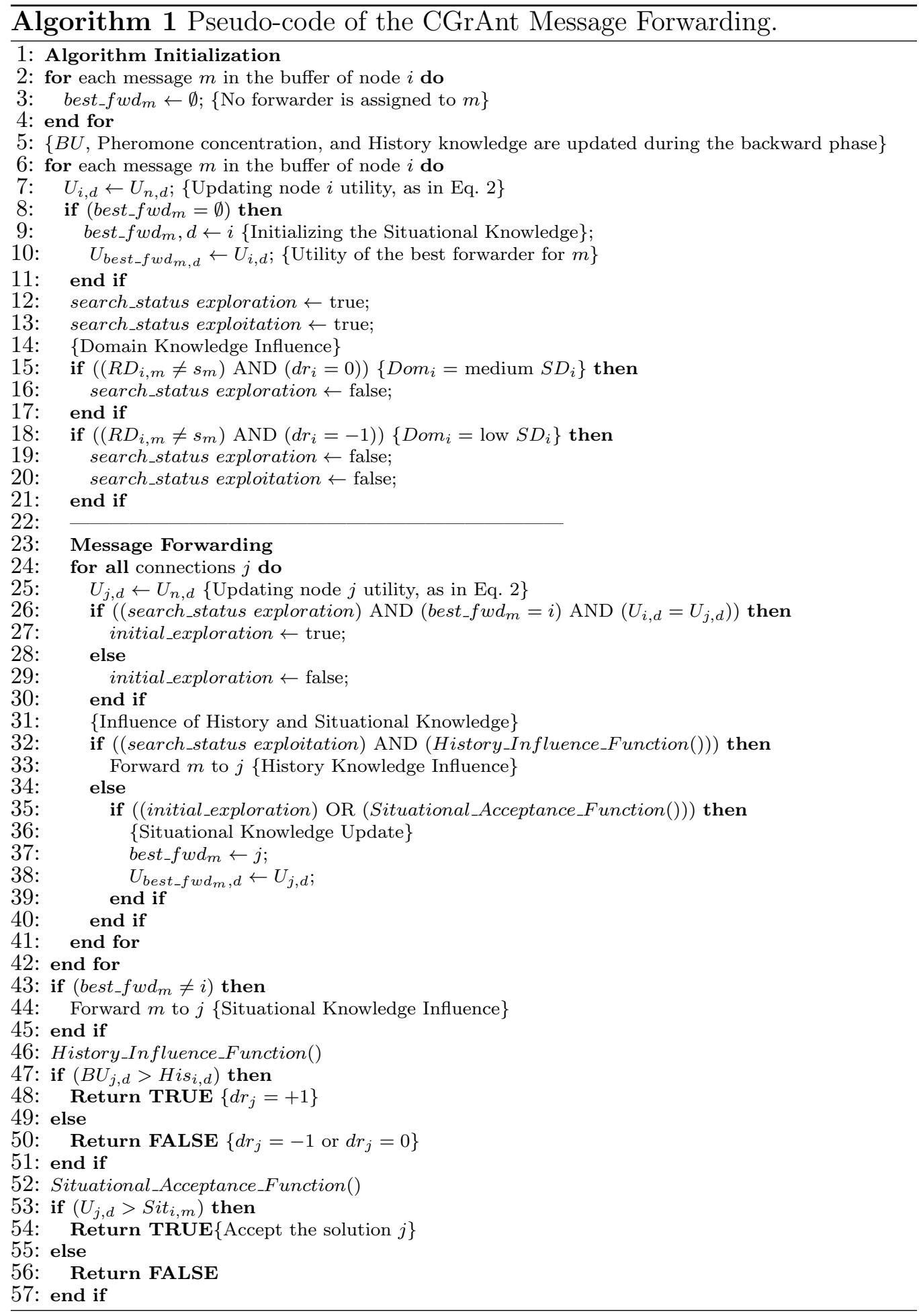


Table 2: Simulation parameters.

\begin{tabular}{|c|c|c|}
\hline Protocol & Setting Parameters & Both scenarios \\
\hline CGrAnt & $\begin{array}{l}\text { Pheromone evaporation rate } \\
\text { Heuristic function } \\
\text { Pheromone concentration } \\
\text { Utility of a node }\end{array}$ & $\begin{array}{c}\mathbf{0 . 1} \\
\left\{\mathbf{S P}_{\mathbf{n}, \mathbf{d}}, D C_{n}, B U_{n, d}, F B_{n}\right\} \\
\left\{|p|, D C_{n}, B U_{n}, \mathbf{D C} \mathbf{C}_{\mathbf{n}}+|\mathbf{p}|\right\} \\
\left\{\text { Heuristic, Pheromone, Heuristic+Pheromone, Heuristic + Pheromone }+\mathbf{R D}_{\mathbf{i}, \mathbf{m}}\right\}\end{array}$ \\
\hline PROPHET & $\begin{array}{l}\text { Hop-count field (hops) } \\
\qquad P_{\text {Inic }} \\
\gamma \\
\text { One time unit Unit }(\mathrm{s}) \\
\varphi\end{array}$ & $\begin{array}{c}11 \\
0.75 \\
0.98 \\
30 \\
0.25\end{array}$ \\
\hline Epidemic & Hop-count field (hops) & 11 \\
\hline Protocol & Communication Parameters & "WD \\
\hline \multirow[t]{2}{*}{ All } & \multirow{2}{*}{$\begin{array}{c}\text { Number of nodes }(\mathrm{N}) \\
\text { Area }\left(\mathrm{m}^{2}\right) \\
\text { Nodes speed }(\mathrm{m} / \mathrm{s}) \\
\text { Waiting time }(\mathrm{s}) \\
\text { Traffic generation rate }(\mathrm{s}) \\
\text { Message TTL }(\mathrm{min}) \\
\text { Nodes buffer }(\mathrm{MB}) \\
\text { Simulation time }(\mathrm{s}) \\
\text { Warm up period }(\mathrm{s}) \\
\text { Communication range }(\mathrm{m}) \\
\text { Transmission rate }(\mathrm{Mbps}) \\
\text { Number of simulations }\end{array}$} & $\begin{array}{c}\mathbf{3 3 9} \\
\mathbf{1 0 , 0 0 0} \times \mathbf{8 , 0 0 0} \\
{[\mathbf{0 . 8 , 1 . 4}] \text { (pedestrian), }[\mathbf{7 . 0 , 1 0 . 0}] \text { (car and bus) }} \\
\mathbf{3 0 0 - 5 0 0}(\mathrm{H}), \mathbf{1 0 - 3 0} \text { (bus) } \\
\mathbf{1 0 0 - \mathbf { 1 5 0 }} \\
\{300,600,900,1200,1500, \mathbf{1 8 0 0}, 2100\} \\
\{4,6,8, \mathbf{1 0}, 12,14,16\}\end{array}$ \\
\hline & & $\begin{array}{c}\mathbf{8 0 0 , 0 0 0} \\
\mathbf{5 , 0 0 0} \\
\mathbf{1 0} \text { (Bluetooth Devices), } 100 \text { (WiFi Devices) } \\
\mathbf{2} \text { (Bluetooth Devices), } 10 \text { (WiFi Devices) } \\
\mathbf{3 0}\end{array}$ \\
\hline
\end{tabular}

Table 3: Additive Analysis of the CGrAnt's Components

\begin{tabular}{|l|c|c|}
\hline Configuration & (PoI $\|$ WD) Message Delivery \% & (PoI $\|$ WD) Message Redundancy \\
\hline \hline 1. Heuristic Function & $46.26 \pm 0.18 \| 53.38 \pm 0.60$ & $18.36 \pm 0.08 \| 85.87 \pm 0.94$ \\
\hline 2. Pheromone Concentration + RD & $48.61 \pm 0.20 \| 54.97 \pm 0.61$ & $15.97 \pm 0.08 \| 68.62 \pm 0.96$ \\
\hline 3. Domain Knowledge 1 & $49.40 \pm 0.21 \| 64.26 \pm 0.63$ & $15.11 \pm 0.09 \| 43.18 \pm 0.41$ \\
\hline 4. Situational Knowledge & $56.70 \pm 0.27 \| 61.04 \pm 0.68$ & $6.86 \pm 0.04 \| 10.87 \pm 0.11$ \\
\hline 5. History Knowledge & $58.93 \pm 0.19 \| 63.70 \pm 0.69$ & $10.37 \pm 0.05 \| 19.04 \pm 0.16$ \\
\hline 6. Domain Knowledge 2 & $58.93 \pm 0.19 \| 63.19 \pm 0.72$ & $10.37 \pm 0.05 \| 12.43 \pm 0.12$ \\
\hline
\end{tabular}

Table 4: Eliminatory Analysis of the CGrAnt's Components

\begin{tabular}{|l|c|c|}
\hline CGrAnt & (PoI $\|$ WD) Message Delivery \% & (PoI $\|$ WD) Message Redundancy \\
\hline \hline All components & $58.93 \pm 0.19 \| 63.19 \pm 0.72$ & $10.37 \pm 0.05 \| 12.43 \pm 0.12$ \\
\hline Without Domain Knowledge & $57.90 \pm 0.22 \| 62.30 \pm 0.67$ & $10.45 \pm 0.04 \| 23.90 \pm 0,28$ \\
\hline Without Situational Knowledge & $53.54 \pm 0.22 \| 64.23 \pm 0.64$ & $15.74 \pm 0.07 \| 43.32 \pm 0,43$ \\
\hline Without History Knowledge & $56.70 \pm 0.27 \| 61.04 \pm 0.68$ & $6.86 \pm 0.04 \| 10.87 \pm 0.11$ \\
\hline
\end{tabular}




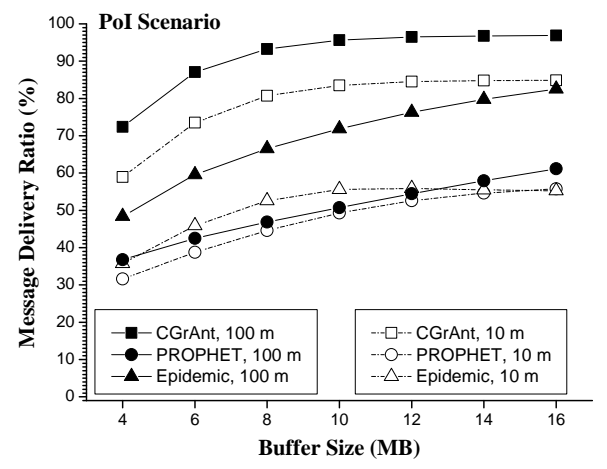

(a) Delivery Ratio

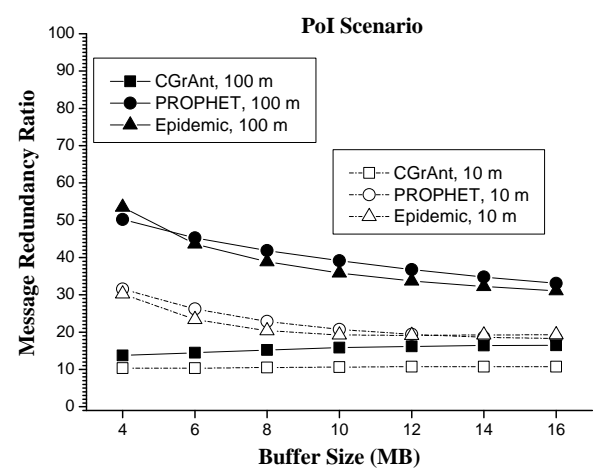

(b) Redundancy Ratio

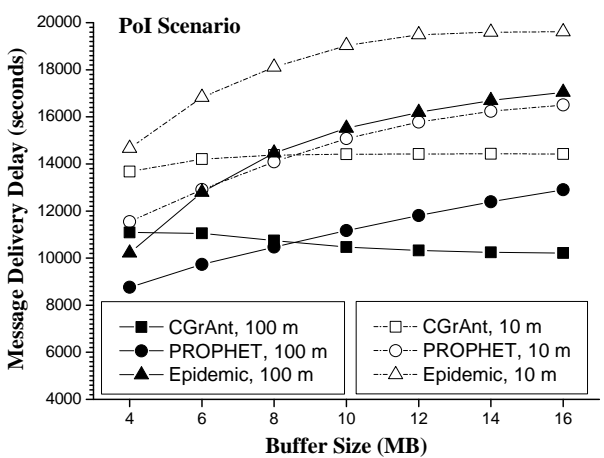

(c) Delivery Delay

Figure 6: Protocols' performance over different buffer sizes - PoI scenario. 


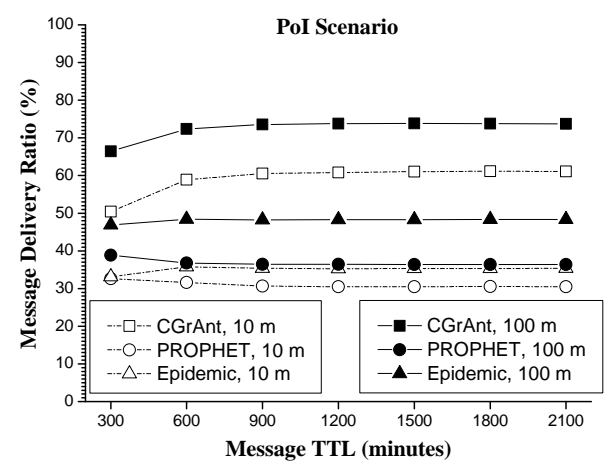

(a) Delivery Ratio

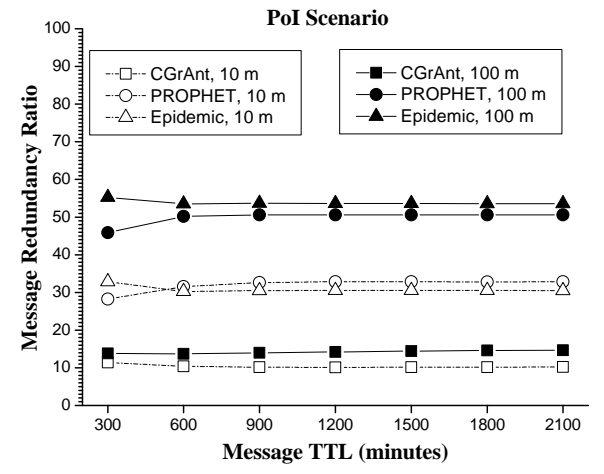

(b) Redundancy Ratio

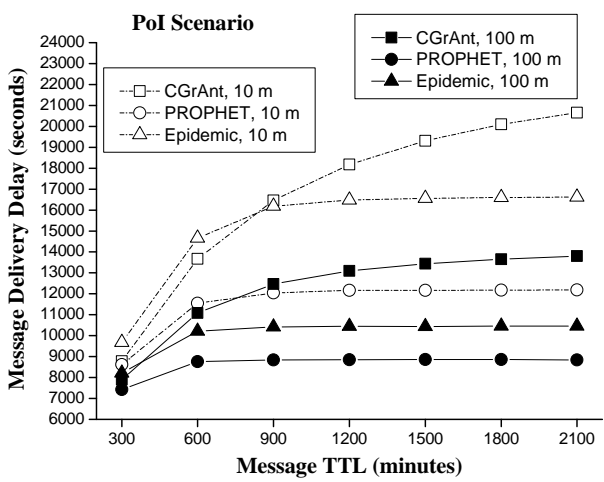

(c) Delivery Delay

Figure 7: Protocols' performance over different message TTLs - PoI scenario. 


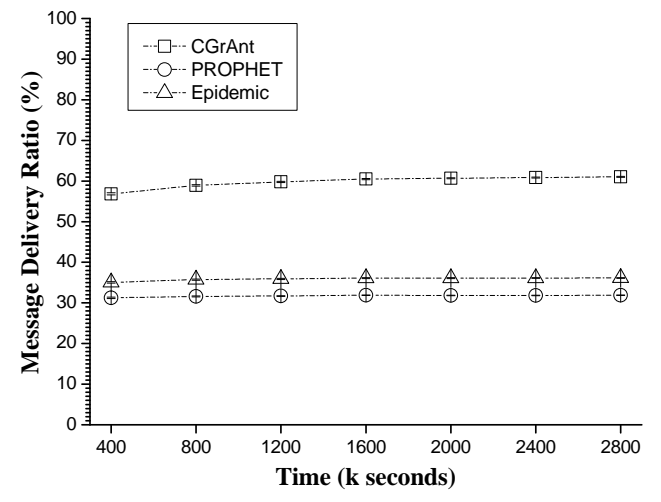

(a) Delivery Ratio - PoI scenario

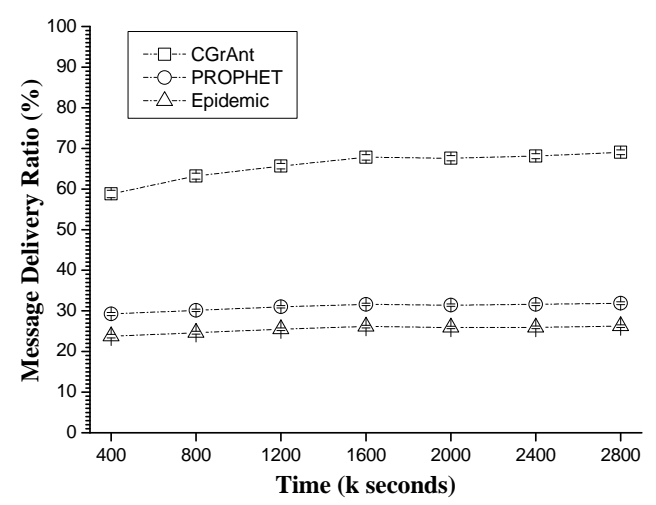

(b) Delivery Ratio - WD scenario

Figure 8: Message Delivery Ratio over Different Simulation Time. 
Table 5: Storage cost over different simulation time

\begin{tabular}{|c|c|c|c|c|c|c|c|}
\hline $\begin{array}{l}\text { Protocols/ } \\
\text { Simulation Time }\end{array}$ & $\begin{array}{c}\text { Registers/Bytes } \\
\text { (100k sec.) }\end{array}$ & $\begin{array}{c}\text { Registers/Bytes } \\
\text { (200k sec.) }\end{array}$ & $\begin{array}{c}\text { Registers/Bytes } \\
\text { (300k sec.) }\end{array}$ & $\begin{array}{c}\text { Registers/Bytes } \\
\text { (400k sec.) }\end{array}$ & $\begin{array}{c}\text { Registers/Bytes } \\
(500 \mathrm{k} \mathrm{sec} .)\end{array}$ & $\begin{array}{c}\text { Registers/Bytes } \\
\text { (600k sec.) }\end{array}$ & $\begin{array}{c}\text { Registers/Bytes } \\
\text { (700k sec.) }\end{array}$ \\
\hline $\begin{array}{l}\text { CGrAnt } \\
F E_{i, d}\end{array}$ & $93.97 / 1,127.65$ & $116.01 / 1,392.17$ & $126.73 / 1,520.74$ & $131.67 / 1,580.06$ & $134.79 / 1,617.43$ & $136.51 / 1,638.17$ & $137.54 / 1,650.51$ \\
\hline$D E_{i, d}$ & $93.97 / 2,255.31$ & $116.01 / 2,784$ & $126.73 / 3,041.49$ & $131.67 / 3,160.11$ & $134.79 / 3,234.86$ & $136.51 / 3,276.34$ & $137.54 / 3,301.03$ \\
\hline$D C_{i}$ & $2 / 16$ & $2 / 16$ & $2 / 16$ & $2 / 16$ & $2 / 16$ & $2 / 16$ & $2 / 16$ \\
\hline$\overline{P T_{i}}$ and $\overline{M S_{i}}$ & $2 / 16$ & $2 / 16$ & $2 / 16$ & $2 / 16$ & $2 / 16$ & $2 / 16$ & $2 / 16$ \\
\hline$S D_{j}$ & $90.82 / 1,453.14$ & $116.01 / 1,856.23$ & $126.73 / 2,027.66$ & $131.67 / 2,106.74$ & $134.79 / 2,156.57$ & $136.51 / 2,184.23$ & $137.54 / 2,200.69$ \\
\hline$U_{\text {best_f }_{f} f d_{m}}$ & $22.55 / 360.8$ & $43.64 / 698.29$ & $62.99 / 1,007.89$ & $81.72 / 1,307.54$ & $98.99 / 1,583.77$ & $115.63 / 1,850.06$ & $131.94 / 2,111.09$ \\
\hline Pheromone Table & $9.56 / 229.54$ & $17.76 / 426.34$ & $23.94 / 574.63$ & $28.76 / 690.17$ & $32.56 / 781.54$ & $35.96 / 862.97$ & $38.74 / 929.66$ \\
\hline$B U_{i, d}$ & $8.58 / 102.94$ & $16.1 / 193.2$ & $22.04 / 264.43$ & $26.76 / 321.09$ & $30.54 / 366.51$ & $33.88 / 406.54$ & $36.74 / 440.91$ \\
\hline$B U_{J, d}^{i}$ & $9.56 / 153.03$ & $17.76 / 284.23$ & $23.94 / 383.09$ & $28.76 / 460.11$ & $32.56 / 521.03$ & $35.96 / 575.31$ & $38.74 / 619.77$ \\
\hline $\begin{array}{l}\text { PROPHET } \\
\text { Delivery Predictability } \\
\text { List }\end{array}$ & $139 / 2,224$ & $139 / 2,224$ & $139 / 2,224$ & $139 / 2,224$ & $139 / 2,224$ & $139 / 2,224$ & $139 / 2,224$ \\
\hline
\end{tabular}

